\title{
On the Design of MAC Protocols for Multi-Packet Communication in IEEE 802.11 Heterogeneous Networks Using Adaptive Antenna Arrays
}

\author{
Fulvio Babich, Senior Member, IEEE, Massimiliano Comisso, Member, IEEE, \\ Alessandro Crismani, and Aljoša Dorni, Member, IEEE
}

\begin{abstract}
This paper discusses the design requirements for enabling multiple simultaneous peer-to-peer communications in IEEE 802.11 asynchronous networks in the presence of adaptive antenna arrays, and proposes two novel access schemes to realize multipacket communication (MPC). Both presented solutions, which rely on the information acquired by each node during the monitoring of the network activity, are suitable for distributed and heterogeneous scenarios, where nodes equipped with different antenna systems can coexist. The first designed scheme, called threshold access MPC (TAMPC), is based on a threshold on the load sustainable by the single-node, while the second protocol, called signal-to-interference ratio (SIR) access MPC (SAMPC), is based on an accurate estimation of the SIR and on the adoption of low density parity check codes. Both protocols, which are designed to be backward compatible with the 802.11 standard, are numerically tested in realistic scenarios. Furthermore, the performance of the two schemes is compared to the theoretical one and to that of the $802.11 \mathrm{n}$ extension in a mobile environment.
\end{abstract}

Index Terms-Distributed networks, wireless communication, access schemes

\section{NOTATION}

$b$

$\mathcal{C}$

$\mathcal{D}$

$\mathrm{d}(i)$

$f_{i, j}(t)$

$G_{i}(\phi)$

$G_{i}^{\mathrm{a}}$

$G_{i}^{\mathrm{n}}$

$\mathrm{I}_{i, j}(t)$

$\mathcal{I}_{i, j}^{0}$

$\mathcal{I}_{i, j}^{0^{\mathrm{a}}}$

$\mathcal{I}_{i, j}^{0^{\mathrm{n}}}$

$\mathcal{I}_{i, j}^{\mathrm{a}}$

$\mathcal{I}_{i, j}^{\mathrm{n}}$

$\mathrm{ID}_{i}$

K
Number of bits used to quantize the SIR.

Set of non-legacy nodes currently active on the multiple communications channel.

Subset of $\mathcal{C}$ containing the active destinations.

Destination of source $i$.

Indicator function of the activity of node $j$ at time $t$ estimated by node $i$.

Antenna gain of node $i$ towards direction $\phi$.

Average antenna gain of node $i$.

Antenna gain of node $i$ in a null.

Interference power at node $j$ and time $t$ estimated by node $i$.

Set of interferers of node $j$ at the beginning of its communication estimated by node $i$.

Subset of $\mathcal{I}_{i, j}^{0}$ of the not suppressed interferers.

Subset of $\mathcal{I}_{i, j}^{0}$ of the suppressed interferers.

Set of current not suppressed interferers of node $j$ estimated by node $i$.

Set of current suppressed interferers of node $j$ estimated by node $i$.

Identifier of node $i$.

Number of samples in azimuth domain.

- F. Babich and M. Comisso are with the Department of Engineering and Architecture, University of Trieste, Via A. Valerio 1034127 , Trieste, Italy.

E-mail: \{babich,mcomisso\}@units.it.

- A. Crismani and A. Dorni are with u-blox, Triest, Italy.

E-mail: \{alessandro.crismani, aljosa.dorni\}@u-blox.com.

Manuscript received 25 Apr. 2014; revised 29 Aug. 2014; accepted 17 Dec. 2014. Date of publication 0 . 0000; date of current version 0.0000.

For information on obtaining reprints of this article, please send e-mail to: reprints@ieee.org, and reference the Digital Object Identifier below.

Digital Object Identifier no. 10.1109/TMC.2014.2385058
Number of iterations of beamforming algorithm.

Access threshold on the number of active communications of node $i$.

Current minimum access threshold on the number of active communications estimated by node $i$.

Number of transmitters estimated by node $i$.

$\begin{array}{ll}l_{i} & \text { Number of transmitters estima } \\ N_{i} & \text { Number of antennas of node } i .\end{array}$

$\mathcal{N} \quad$ Set of all network nodes.

$\mathcal{N}^{-1} \quad$ Subset of $\mathcal{N}$ containing the legacy nodes.

$\mathcal{N}^{\mathrm{n}} \quad$ Subset of $\mathcal{N}$ containing the non-legacy nodes.

$n \quad$ Number of network nodes.

$n_{1} \quad$ Number of legacy nodes.

$n_{\mathrm{n}} \quad$ Number of non-legacy nodes.

$\mathrm{NAV}_{i} \quad$ Network allocation vector of node $i$.

$\mathrm{NCT}_{i} \quad$ Neighboring characteristic table of node $i$.

$P_{i, j}^{\mathrm{r}} \quad$ Power received by node $i$ from node $j$.

$R_{i} \quad$ Code rate used in transmission by node $i$.

$R_{i, j}^{\mathrm{s}}(t) \quad$ Code rate function at node $j$ and time $t$ estimated by node $i$.

$\bar{R}_{i, j}^{\mathrm{s}} \quad$ Sustainable code rate at node $j$ estimated by node $i$.

$\mathcal{R} \quad$ Set of selectable code rates.

$\mathcal{S} \quad$ Subset of $\mathcal{C}$ containing the active sources.

$\mathrm{s}(i) \quad$ Source of destination $i$.

$\operatorname{SIR}_{i, j}(t) \quad$ SIR at node $j$ and time $t$ estimated by node $i$.

$T_{\mathrm{A}} \quad$ Acknowledgment packet transmission time.

$T_{\mathrm{D}} \quad$ DATA packet transmission time.

$T_{\mathrm{f}} \quad$ Time required by a floating point operation.

$T_{i_{\mathrm{n}}} \quad$ Packet transmission time normalized to the slot time for node $i$.

$t_{i} \quad$ Instant of beginning of a reception at node $i$.

$t_{\mathrm{M}}^{\mathrm{S}} \quad$ Computational time required by SAMPC scheme.

$t_{\mathrm{M}}^{\mathrm{T}} \quad$ Computational time required by TAMPC scheme. 
$t_{\mathrm{P}} \quad$ Computational time due to antenna processing.

$\eta_{i, j} \quad$ Probability of activity of node $j$ on the multiple communications channel estimated by node $i$.

$\sigma_{\mathrm{DOA}} \quad$ Standard deviation of the error on the estimated direction of arrival.

$\sigma_{\text {RSSI }} \quad$ Standard deviation of the error on the received signal strength indicator.

$\sigma_{\phi} \quad$ Channel angular spread.

$\phi \quad$ Azimuth angle.

$\phi_{i, j} \quad$ Direction of arrival of node $j$ at node $i$.

$\tau \quad$ Slot time

\section{INTRODUCTION}

$\mathrm{T}$ HE current leading technology for low-cost wireless access is based on the family of IEEE 802.11x medium access control (MAC) and physical (PHY) layer protocols [1]. However, still nowadays the support for high-throughput applications, such as remote desktop and high-definition video streaming, represents a challenging task in the presence of multiple concurrent users. Within this field, the capacity increase achievable using advanced antenna systems is a widely investigated issue, since from the approval of the 802.11 legacy standard [2], [3], [4], [5], [6]. Accordingly, the task group $\mathrm{n}$ has developed the 802.11n amendment [7], which uses multi-antenna systems for increasing the data rate of the single link, but maintains the access limited to just one user at a time. To deal with this limitation, the benefits of advanced antenna systems for supporting multiple simultaneous communications have been explored by theoretical [8], [9], [10], [11], [12], [13], [14], [15], [16], [17], [18], and numerical/experimental studies [19], [20], [21], [22], [23], [24], [25], [26], [27], [28], [29], [30], [31], [32], [33], [34], [35]. In this context the research efforts are currently focused on three main topics: the simultaneous transmission of different packets from a unique source to multiple destinations, known as multi-packet transmission (MPT), the simultaneous reception from multiple sources by a unique destination, known as multi-packet reception (MPR), and the concurrent communication between different node pairs, known as multi-packet communication (MPC). In MPT/MPR scenarios the access must be synchronized, since all transmissions must begin at the same time instant [8], [11]. By contrast, in MPC scenarios, the access can be asynchronous, since each source may start its transmission independently of the instants chosen by the other sources [12], [13]. Among these three strategies, the first that has been deployed is MPT, whose functionalities, relying on multi-user multiple-input multiple-output (MU-MIMO) technology, are supported in long-term evolution (LTE) Release 8 and finalized in the 802.11ac standard for downlink operations between the access point (AP) and the users [36].

MPT/MPR are widely considered suitable for centralized scenarios, where the synchronization is guaranteed by the AP [19], [20], [21], [22], [23], and [25], while, in distributed scenarios, the synchronization may be enabled by properly modifying the request-to-send/clear-to-send (RTS/CTS) handshake of the distributed coordination function (DCF) [24]. However, the results in [13] show that, for distributed scenarios, MPC is more suitable, since an asynchronous access can provide a higher throughput than a synchronous one. On the other hand, smart antenna systems cannot be completely exploited in a distributed scenario, since the distribution of the active transmitters can change when a source-destination communication is already in progress [18]. Despite that, a centralized control can be sometimes not realizable or disadvantageous and hence several MPC extensions for the 802.11 DCF have been presented [26], [27], [28], [29], [30], [31], [32], [33], [34], [35]. Among these MPC proposals, three main aspects should be considered [33], [34], [35]. Firstly, the 802.11 backward compatibility, which may be compromised by even minor modifications of the access rules that may inhibit the communications of the nodes relying on the standard access mechanism. Secondly, the heterogeneity of the scenario, which concerns the coexistence of nodes with different antenna systems and the capability of preventing that the more advanced nodes acquire all the resources at the expense of the other nodes. Finally, the third aspect concerns the ability of the access scheme to provide position and traffic information to each node, in order to approach as much as possible the performance of a centralized network [18].

This paper deals with these three issues by discussing the main requirements that should be satisfied by an 802.11 MPC extension using adaptive arrays. Two novel MAC protocols are designed according to these requirements. The first protocol, called threshold access MPC (TAMPC), adopts an access policy based on a threshold on the sustainable load, which depends on the single-node antenna capabilities and has been analytically derived in [13]. The second protocol, called signal-to-interference ratio (SIR) access MPC (SAMPC), relies on a specific parameter, called sustainable code rate, whose calculation is based on an accurate estimation of the SIR and on the selected channel code rate. The two developed schemes are tested on a wide variety of network scenarios using a realistic simulation platform and considering different performance figures. This work represents an exhaustive numerical completion of the preliminary studies in [37], [38]. In particular, with respect to [37], [38], this paper introduces improved access mechanisms, additionally discussing their computational cost, their sensitivity to deafness, hidden terminal, mobility, topology, and estimation errors, and their performance with respect to the theory and the $802.11 \mathrm{n}$ extension. The aim is to provide MAC layer solutions managing MPC scenarios simultaneously characterized by heterogeneity, asynchrony, and backward compatibility, since the joint consideration of these issues represents a relevant but partly unexplored topic.

The paper is organized as follows. Section 2 presents the literature overview. Section 3 introduces the network scenario. Section 4 identifies the MAC design strategy. Sections 5 and 6 describe the proposed TAMPC and SAMPC protocols, respectively. Section 7 presents the computational analysis. Section 8 reports and discusses the numerical results. Section 9 summarizes the most relevant conclusions.

\section{Related Work}

The use of directional radiation patterns has been identified in [2] as a solution for alleviating the scalability limitations of ad-hoc networks. This may be achieved by decreasing the 
beamwidth according to the increase of the number of nodes [3], by reducing the side lobe level [4], by combining the effect of spatial reuse and mobility to statistically decrease the interference power [5], or by effectively exploiting the really available degrees of freedom of a multi-antenna node [6].

The wide diffusion of the 802.11 standard has further focused the theoretical research on MPT/MPR/MPC towards 802.11 networks [8], [9], [10], [11], [12], [13], [14], [15], [16], [17], [18]. In particular, the impact of the MPR collision model on the throughput of a random access network is explored in [8]. A cross-layer analysis of the 802.11 DCF in the presence of directional communications and realistic channel conditions is proposed in [9]. Mathematical models for 802.11 multi-packet scenarios have been developed in [10], which focuses on the impact of a directional virtual carrier sensing (DVCS) mechanism, and in [11], which investigates the influence of the channel-antenna characteristics on the throughput-delay performance. The asynchronous access is explored in [12], which proposes a cross-layer redesign of a carrier sensing multiple access network, and in [13], which develops a theory for evaluating the throughput of an 802.11 MPC network that is extended in [14], [15] using the collision model of [16]. A detailed capture analysis of an MPR receiver having interference cancellation capabilities is presented in [17]. In [18] the author observes that all the conditions required to exploit adaptive arrays in wireless networks can be completely satisfied only in a centralized MPT/MPR scenario. A MAC protocol for enabling MPR at 802.11 APs equipped with multi-beam antennas is proposed in [19] by adopting both space-division and time-division multiplexing. Experimental MPR testbeds are implemented in [20], where space-time multiplexing and conflict graphs are used to schedule the transmissions, in [21], where the access is managed by counting the active communications, and in [22], where the MAC protocol is designed accounting for the most suitable antenna configuration. Asynchronous 802.11 MPR schemes are developed in [23], which adopts space-time coding techniques, and in [24], which exploits spatial multiplexing. A rate-adaptation algorithm for MPR networks is designed and experimentally tested in [25].

Even if [18] identifies a fundamental issue that cannot be completely satisfied in a distributed scenario, namely the preservation of the active nodes' distribution at the beginning of a communication for its entire duration, several wireless networks have to operate in ad-hoc mode due to the lack of a coordinating infrastructure. Hence, together with the MPT/MPR proposals, several MAC protocols have been developed for distributed MPC networks [26], [27], [28], [29], [30], [31], [32], [33], [34], [35]. In particular, [26] adopts the DVCS mechanism for increasing the capacity in the presence of adaptive arrays, while [27] proposes a cooperative approach for suppressing interference. Multi-antenna extensions for slotted Aloha and for the 802.11 basic access are presented in [28]. A centralized MAC protocol, from which a distributed version is derived, is proposed in [29] for ad-hoc networks with MIMO links. A multi-hop RTS approach is presented in [30], moving from the typical problems related to the use of directional antennas in 802.11 networks: deafness and hidden terminal. A MAC protocol adopting transmit/ receive beamforming in multipath environment is designed in [31]. In [32] multiple communications are managed using a circular directional RTS transmission that notifies the availability of the antenna beams. MPC and interoperability between directional and omnidirectional antennas are achieved in [33], by introducing the concept of DVCS, and in [34], by generalizing the 802.11 clear channel assessment function. An excellent MPC scheme for heterogeneous MIMO networks, called $802.11 n^{+}$, has been developed and experimentally tested in [35].

This overview shows that the research on MPC addresses two main issues: theoretical modeling and protocol design. Within this second field, except from [34], [35], the proposed MPC schemes are not validated in completely heterogeneous scenarios, where all nodes can have different antenna systems. Moreover, many schemes introducing modifications in the RTS/CTS handshake [26], [29], [35], novel control frames [27], [30], [31] [32], or tones [28], are often not explicitly tested in mixed scenarios including nodes following the legacy standard and others implementing the novel protocol, thus making difficult to directly infer the backward compatibility of the conceived solutions. Hence, the design of an MPC scheme for managing an asynchronous and heterogeneous scenario, and developed moving from the constraint of backward compatibility, still represents an open issue. The MAC protocols described in this paper aim to match this objective, hence providing numerically validated strategies for the design of backward compatible MPC solutions for distributed, asynchronous, and heterogeneous 802.11 networks.

\section{SCENARIO DESCRIPTION}

The considered scenario is characterized by two main features: asynchrony and heterogeneity. Being the 802.11 DCF a slotted algorithm, the asynchrony does not refer to the lack of clock synchronization, but to the possibility that each source starts its transmission, at the beginning of a slot, regardless of the slots chosen by the other sources. Thus, a slotted access is assumed and the asynchrony implies the absence of policies for grouping the contending sources [26], [27], [28], [29], [30], [31], [32], [33], [34], [35]. The heterogeneity stems from the coexistence of nodes that differ in terms of antenna system characteristics, channel conditions, and position inside the topology. In fact, even if a network consists of nodes with identical antenna systems, it can be in practice heterogeneous, because the interference suppression capabilities of the single station depend also on the reciprocal positions of the nodes. In a synchronoushomogeneous scenario with packets of equal length, a collision represents a situation in which all involved packets are lost, since the transmissions are perfectly superimposed because of the synchronization (all bits are corrupted). In the other three cases (homogeneous-asynchronous, heterogeneous-synchronous, heterogeneous-asynchronous), different levels of corruption may be experienced because of the heterogeneity of the antenna systems and/or the asynchrony, thus determining the loss of only a subset of the collided packets. This implies that in a heterogeneous-asynchronous network the consequences of a collision must be 
evaluated locally, since they depend on the individual characteristics of the involved nodes and on the evolution of each SIR.

The managed 802.11 network includes a set $\mathcal{N}$ of $n$ nodes, where the generic node $i(\in \mathcal{N})$ has an antenna system of $N_{i}(\geq 1)$ elements. This set is partitioned in two subsets: the subset $\mathcal{N}^{1}$, containing $n_{1}$ legacy nodes having $N_{i}=1$, and the subset $\mathcal{N}^{\mathrm{n}}$, containing $n_{\mathrm{n}}=n-n_{\mathrm{l}}$ non-legacy nodes having $N_{i}>1$. Precisely, the term 'legacy' identifies a singletransceiver node equipped with a unique antenna and following the 802.11 DCF rules, while the term 'non-legacy' identifies a dual-transceiver multi-antenna node, where the first transceiver is equipped with a unique antenna and the second transceiver with an adaptive array of $N_{i}-1$ elements. Denoting the azimuth angle as $\phi$, this second transceiver can generate a receiving radiation pattern $G_{i}(\phi)$, having the main lobe steered towards the desired direction and the nulls placed towards the interferers [39], which can be characterized by the average gain $G_{i}^{\mathrm{a}}$ and the null gain $G_{i}^{\mathrm{n}}(<<1)$ [11]. The first transceiver is introduced for backward compatibility purposes, while the second one to enable MPC among the non-legacy nodes (details will be clarified in the following sections). The load sustainable by such MPC scenario may be modeled, in the homogeneous case, by the number of transmissions $L_{\mathrm{t}}$ that allows a node to access the medium without destroying the ongoing communications [11], [13]. In the heterogeneous case, each node $i$ has its own $L_{\mathrm{t}_{i}}$ value. If $i$ is a legacy node, then $L_{\mathrm{t}_{i}}=0$, because this node can correctly receive a packet only if no other communications are active, while, if $i$ is a non-legacy node, then $L_{\mathrm{t}_{i}}$ depends on the channel-antenna characteristics and on the network topology [6], [11].

The characteristics of the involved nodes suggest two possible MPC approaches for extending the 802.11 DCF: one based on the resumptive parameter $L_{\mathrm{t}_{i}}$, and another one based on the estimation of the SIR. The first approach summarizes the channel-antenna characteristics using an averaged quantity that may be calculated offline according to the theory of [11], [13] and included in the set of the MAC layer parameters, similarly to the minimum contention window or the retry limit, thus reducing the real-time computational burden. The second approach, instead, considers a real-time estimation of the instantaneous SIR, where the estimation accounts for the antenna parameters and the relative position of a single-node within the network. This second solution may imply a higher computational burden, but may guarantee an access more adherent to the real traffic evolution.

\section{Design Strategy}

\subsection{Requirements and Preliminary Choices}

Regardless of the specific approach, an attractive MPC-DCF extension should fulfill three main requirements. First, the access should be asynchronous, since, in a distributed network, MPC can provide a higher throughput than a synchronous approach [13]. To completely exploit adaptive arrays in this context, a reliable access criterion should consider two main objectives [18]: the acquisition of information on the nodes' activity, and the preservation of the conditions present at the beginning of a transmission for the entire duration of that transmission (since $G_{i}(\phi)$ cannot

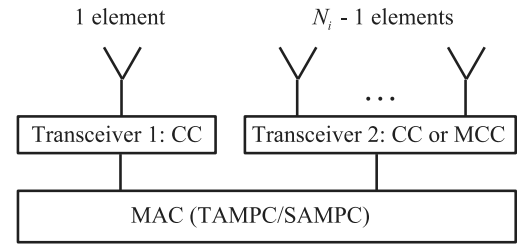

Fig. 1. Non-legacy node: channel usage.

be changed during the communication). While the first objective may be reached by monitoring the medium and by properly exploiting the DCF handshake, the second objective cannot be completely achieved, because of the asynchrony and the lack of coordination [18]. Thus, to enable MPC, a MAC protocol should enable the acquisition of the distribution of the interferers at the beginning of a transmission, estimating the possible evolution of such distribution. The second requirement for an MPC-DCF extension is its backward compatibility. In fact, MPC must be introduced avoiding modifications of the access rules that may be perceived by the legacy nodes. The third requirement concerns the heterogeneity of the scenario. Since 802.11 networks can be extended by adding new nodes and the progresses in antenna miniaturization enable inserting more and more antennas on a device [40], the scenario of the near future may involve devices of different generations, characterized by increasing antenna capabilities. Thus, heterogeneity may represent a plausible aspect of forthcoming networks, where the MAC protocol should prevent nodes having more powerful antenna systems from acquiring all the resources at the expense of the nodes having less powerful antenna systems.

The above requirements suggest some preliminary choices in the design strategy for extending the DCF to MPC.

First, the RTS/CTS access seems preferable to the basic one [26], [27], [29], [30], [31], [32], [33], [34], [35], since the former enables the dissemination of more information concerning each communication. The basic access may be even modified to allow the support of multiple communications [28], but with lower throughput results [18].

The second choice consists in adopting two transceivers and hence two non-overlapping frequency channels (Fig. 1), where the first channel can be used by both legacy and nonlegacy nodes, and represents the common channel (CC), while the second one can be used only by the non-legacy nodes, and represents the multiple communications channel (MCC). Just one communication at a time is allowed on the CC, while multiple simultaneous communications are allowed on the MCC. A legacy node monitors only the CC, while a non-legacy node performs an omnidirectional monitoring on both channels using the two transceivers. The main reason for using two channels is that it allows using modified control packets on the MCC. Non-standard frames cannot be used in a channel shared with the legacy nodes, which are unable to understand the novel formats. Besides, in the standard RTS/CTS frames there are no bits available for including novel information, since all fields are used for standard operations [19].

The third choice concerns the adoption of beamforming only for reception, maintaining all transmissions as omnidirectional. This choice implies, first, that all nodes can receive 
the RTS/CTS packets, thus being informed about the forthcoming communication, and, second, that a busy non-legacy node having lost this RTS/CTS exchange can update its knowledge of the medium occupation once its communication is completed by sensing the omnidirectional DATA and acknowledgement (ACK) transmissions. Hence, a nonlegacy source can decide whether its communication will interfere or not with any existing one, thus avoiding to act as an hidden terminal, and can know if its destination is idle, thus avoiding deafness. Besides, the channel separation combined with the omnidirectional transmissions implies that a legacy source cannot be an hidden terminal for another node. Moreover, to reduce the last possibility of deafness when a legacy source intends to communicate with a non-legacy destination already involved in a communication on the MCC, a priority mechanism is introduced. In this way the request of the legacy node is always detected by the non-legacy node, since the CC is monitored by its first transceiver (Fig. 1), and the communication in the MCC is interrupted to respond to the legacy node. Observe that the other non-legacy node involved in the interrupted communication is aware of the reason of the interruption, since it also monitors the CC, thus the retransmission can be scheduled without modifying the retry counter and the contention window. The impact of this preemptive priority will be studied in Section 8.5. In summary, the combination of RTS/CTS access, dual channel usage, and omnidirectional sensing/ transmission, allows a considerable robustness against the hidden terminal and deafness phenomena, thanks to an exhaustive dissemination of traffic information, thus matching the first objective for exploiting adaptive arrays in a wireless network [18]. Besides, legacy and non-legacy nodes can coexist, since the former operate just on the CC using standard frames, while the latter operate also on the MCC, where the frames can be non-standard and MPC is allowed.

\subsection{Recognition}

The first step of the adopted strategy aims to enable each non-legacy node to distinguish between legacy and nonlegacy nodes preserving backward compatibility. This recognition, which, by using the first transceiver, must be performed on the CC, can be carried out using the More Data field of the frames RTS (RTS[MD]) and CTS (CTS[MD]). The 802.11 standard states that this field, consisting of a unique bit and having a meaning only for centralized operations, must be set equal to zero and ignored by the legacy nodes during the contention period [[1], p. 37]. In the proposed MPC design, instead, this field can be set to one and read even during the contention period by the non-legacy nodes. In particular, when a non-legacy source $i$ has a packet for a destination $\mathrm{d}(i)$ of unknown antenna characteristics, $i$ can communicate to $\mathrm{d}(i)$ its multi-antenna capabilities by setting to one the transmitted RTS[MD] and can infer the (possible) multi-antenna capabilities of $\mathrm{d}(i)$ by reading the received CTS[MD]. Thus, for a communication $i$-d $(i)$, a non-legacy node $\mathrm{d}(i)$ reads the received RTS[MD] and sets to one the transmitted CTS[MD], while, a legacy node $\mathrm{d}(i)$ does not read the RTS[MD] and does not set to one the CTS[MD] (according to the 802.11 rules). Hence, $i$ can be aware whether it is involved in a non-legacy-non-legacy or in a non-legacy-legacy communication.
This recognition process, which is completely transparent for the legacy nodes, being the RTS/CTS standard format unmodified, is used in both proposed protocols. Each communication involving at least a legacy node is entirely performed on the $\mathrm{CC}$, where only one communication at a time is allowed, by following the usual DCF standard rules. The non-legacy-non-legacy communications are instead performed on the MCC, where the access rules relative to the decrease of the backoff counter are properly modified to enable MPC. Therefore, the TAMPC protocol, presented in Section 5, and the SAMPC protocol, presented in Section 6, are described referring to a non-legacy-non-legacy communication $i-\mathrm{d}(i)$.

\section{Threshold Access MPC Protocol}

The TAMPC scheme relies on the threshold $L_{\mathrm{t}_{i}}$, which accounts for the antenna features of a node $i$ [13].

\subsection{Operations in the CC: Single Communication}

Once the RTS/CTS exchange and the recognition are completed, $i$ and $\mathrm{d}(i)$ perform the DATA/ACK exchange on the $\mathrm{CC}$ using the second transceiver equipped with the adaptive array. Hence, $i$ transmits the DATA packet by adding its threshold $L_{\mathrm{t}_{i}}$ and a preamble for enabling $\mathrm{d}(i)$ to estimate the direction of arrival (DOA) of $i$ and to synthesize the receiving pattern. Similarly, once the DATA reception is completed, $\mathrm{d}(i)$ sends the ACK packet by adding its threshold $L_{\mathrm{t}_{\mathrm{d}(i)}}$ and the preamble. Thus, the first transceiver is used on the CC and the second transceiver on the MCC, except for the first packet exchanged with a non-legacy destination (Fig. 1). After this first RTS/CTS/DATA/ACK handshake $i$ and $\mathrm{d}(i)$ have acquired their reciprocal characteristics, which are inserted in a table, called neighboring characteristic table (NCT), having one entry for each node with which a communication has already been performed. More precisely, for the node $i$, the generic entry $j$ of the $\mathrm{NCT}_{i}$ contains the identifier (ID) of $j\left(\mathrm{ID}_{j}\right)$, the threshold $L_{\mathrm{t}_{j}}$, the DOA $\phi_{i, j}$, and the network allocation vector (NAV) of $j\left(\mathrm{NAV}_{j}\right)$. These modifications of the DATA/ACK packets (threshold and preamble) are not perceived by the legacy nodes because, after the RTS/CTS exchange, they set their NAVs and turn off their radios for the time specified in the duration field. A non-legacy node $k \neq i, \mathrm{~d}(i)$, instead, can monitor the $i$-d $(i)$ DATA/ACK exchange to acquire the parameters for the entries $i$ and $\mathrm{d}(i)$ of its $\mathrm{NCT}_{k}$.

\subsection{Operations in the MCC: Multiple Communications}

When a non-legacy source $i$ has a packet for an already recognized non-legacy destination $\mathrm{d}(i)$, the two nodes communicate on the MCC. As in the DCF, $i$ generates the backoff and monitors the medium (Fig. 2). To this aim, $i$ estimates the DOAs $\phi_{i, 1}, \ldots, \phi_{i, l_{i}}$ of the $l_{i}$ active transmitters. Since the scenario is asynchronous, the estimation $l_{i}$ can include RTS, CTS, DATA, and ACK transmissions. To properly exploit this information, $i$ has to take into account that, since an array with $N_{i}-1$ elements may allow estimating up to $N_{i}-1$ transmitters, this estimation can be considered reliable only for $l_{i} \leq N_{i}-2$, since the estimation $l_{i}=N_{i}-1$ is 


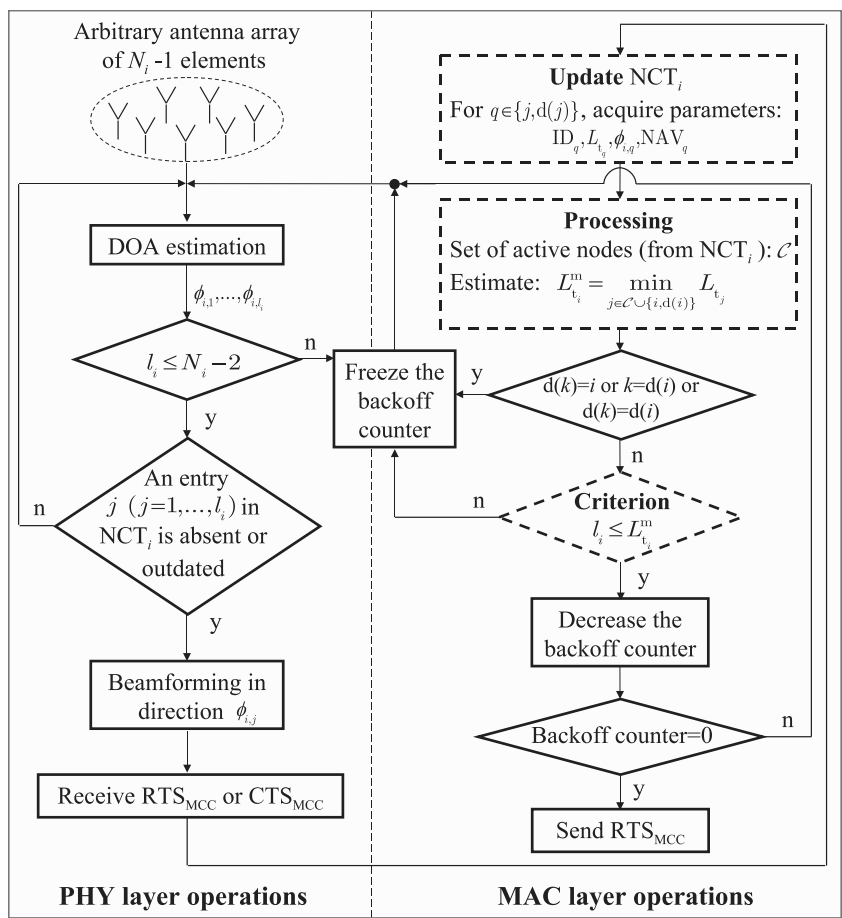

Fig. 2. TAMPC: sensing and backoff decrease rules in the MCC for node $i$.

provided also when the real value of active transmitters in the MCC is larger than $N_{i}-1$. Therefore, if $l_{i}=N_{i}-1$, the MCC is considered busy and $i$ freezes its backoff counter. Instead, if $l_{i} \leq N_{i}-2, i$ examines the $\mathrm{NCT}_{i}$ for each $j=1, \ldots, l_{i}$ to verify if the entry relative to $\phi_{i, j}$ is complete or lacks in the corresponding $\mathrm{ID}_{j}, L_{\mathrm{t}_{j}}$, or has a $\mathrm{NAV}_{j}$ equal to zero. If each entry is complete, $i$ continues to monitor the channel, while, if an entry $j$ is not complete, the packet incoming from $\phi_{i, j}$ is received. To this purpose, $i$ runs its beamforming algorithm by setting $\phi_{i, j}$ as the desired direction and all the other estimated DOAs as the undesired directions. The received packet is then delivered from the PHY to the MAC layer. If this packet is relative to a communication $k-\mathrm{d}(k)$, the contained information is used by $i$ to update the $\mathrm{NCT}_{i}$. Precisely, the $\mathrm{RTS}_{\mathrm{MCC}} / \mathrm{CTS}_{\mathrm{MCC}}$ packets sent on the MCC are extended versions of the RTS/CTS standard packets that contain the IDs, the thresholds, the NAVs relative to $k$ and $\mathrm{d}(k)$, and the preambles for the antenna processing operations. Observe that, since in the MCC the legacy nodes are not present, the structure of the frames can be modified. If the sensed packet has $i$ as its destination $(\mathrm{d}(k)=i)$ or involves the destination of $i$ $(k=\mathrm{d}(i)$ or $\mathrm{d}(k)=\mathrm{d}(i)), i$ freezes its backoff counter. Otherwise, $i$ updates the current threshold as:

$$
L_{\mathrm{t}_{i}}^{\mathrm{m}}=\min _{j \in \mathcal{C} \cup\{i, \mathrm{~d}(i)\}} L_{\mathrm{t}_{j}},
$$

where, using the notation introduced in Section 3, the set $\mathcal{C}=\left\{p \in \mathcal{N}^{\mathrm{n}} \mid \mathrm{NAV}_{p}>0\right\}$ contains the non-legacy nodes involved in a communication on the MCC (having a NAV different from zero in the $\mathrm{NCT}_{i}$ ). If $l_{i} \leq L_{\mathrm{t}_{i}}^{\mathrm{m}}$, the backoff counter can be decreased, since the potential communication $i$ - $\mathrm{d}(i)$ is sustainable and does not destroy the current receptions. Otherwise, the backoff is frozen. This monitoring operation continues until the backoff counter reaches the zero value and the $\mathrm{RTS}_{\mathrm{MCC}} / \mathrm{CTS}_{\mathrm{MCC}} / \mathrm{DATA} / \mathrm{ACK}$ handshake begins.

The TAMPC protocol has the advantage of enabling MPC by relying on a unique parameter that can be analytically derived [11], [13]. Hence, the real-time calculations required by this scheme are substantially limited to DOA estimation and beamforming. Besides, since the access is based on an average quantity that is selected to preserve the active communications, the TAMPC scheme mainly adopts a conservative approach.

\section{SIR ACCESS MPC (SAMPC) PROTOCOL}

With respect to the TAMPC scheme, the SAMPC protocol enables MPC by relying on the instantaneous SIR, which is an indicator more adherent to the real network behavior in terms of antenna pattern, topology, and traffic. The novelty of the SAMPC protocol lies in an accurate estimation of the SIR and of the transmission result at the access stage. This is made feasible by the introduction of efficient codes, such as the low density parity check (LDPC) codes, already allowed in the $802.11 \mathrm{n}$ extension. In general, the use of a channel encoder is advantageous in every asynchronous multi-user system, where the maintenance of the SIR below a given threshold represents a rather difficult task, unless one accepts a really conservative approach and the resulting throughput reduction. In fact, the channel encoder, and the relative interleaver, enable the adoption of a more aggressive approach by allowing one to accept occasionally collided slots within the correction limits of the used code. In particular, the choice of LDPC codes has two main advantages. First, they are more efficient than the convolutional ones adopted in the 802.11 extensions. Second, the performance of efficient codes, such as the LDPC codes can be reliably modeled adopting a threshold approach, based on the sustainable rate [41], which enables a fast and reliable estimation of the success/failure of a transmission attempt.

\subsection{Operations in the CC: Single Communication}

Once $i$ and $\mathrm{d}(i)$ have been reciprocally recognized, $i$ sends, using the second transceiver, the DATA packet, which contains the antenna parameters $N_{i}, G_{i}^{\mathrm{a}}$ (average gain), $G_{i}^{\mathrm{n}}$ (average gain in a null), the power $P_{i, \mathrm{~d}(i)}^{\mathrm{r}}$ received from $\mathrm{d}(i)$, and a preamble to enable $\mathrm{d}(i)$ to estimate the DOA $\phi_{\mathrm{d}(i), i}$ and to synthesize the receiving pattern $G_{\mathrm{d}(i)}(\phi)$ [39]. Since the transmissions are omnidirectional, $P_{i, \mathrm{~d}(i)}^{\mathrm{r}}$ can be inferred from the received signal strength indicator (RSSI), which is already implemented in the commercially available wireless cards [42]. Similarly, the ACK of $\mathrm{d}(i)$ includes $N_{\mathrm{d}(i)}, G_{\mathrm{d}(i)}^{\mathrm{a}}$, $G_{\mathrm{d}(i)}^{\mathrm{n}}, P_{\mathrm{d}(i), i}^{\mathrm{r}}$, and the preamble to enable $i$ to evaluate $\phi_{i, \mathrm{~d}(i)}$ and $G_{i}(\phi)$. Since a unique omnidirectional transmission is allowed in the CC, also a non-legacy node $k \neq i, \mathrm{~d}(i)$, sensing the current DATA/ACK exchange, can acquire the parameters $N_{i / \mathrm{d}(i)}, G_{i / \mathrm{d}(i)}^{\mathrm{a}}, G_{i / \mathrm{d}(i)}^{\mathrm{n}}, P_{i, \mathrm{~d}(i)}^{\mathrm{r}}, P_{\mathrm{d}(i), i}^{\mathrm{r}}$ and can estimate the two DOAs $\phi_{k, i / \mathrm{d}(i)}$ and the two powers $P_{k, i / \mathrm{d}(i)}^{\mathrm{r}}$. Besides, the node $k$ also stores $\mathrm{NAV}_{i}$ and $\mathrm{NAV}_{\mathrm{d}(i)}$. These data are inserted by $k$ into the $\mathrm{NCT}_{k}$, having, as in the TAMPC scheme, one entry for each sensed node $j$, but now containing $N_{j}, G_{j}^{\mathrm{a}}, G_{j}^{\mathrm{n}}, \mathrm{NAV}_{j}, \phi_{i, j}$, and all powers $P_{j, p}^{\mathrm{r}}$ 


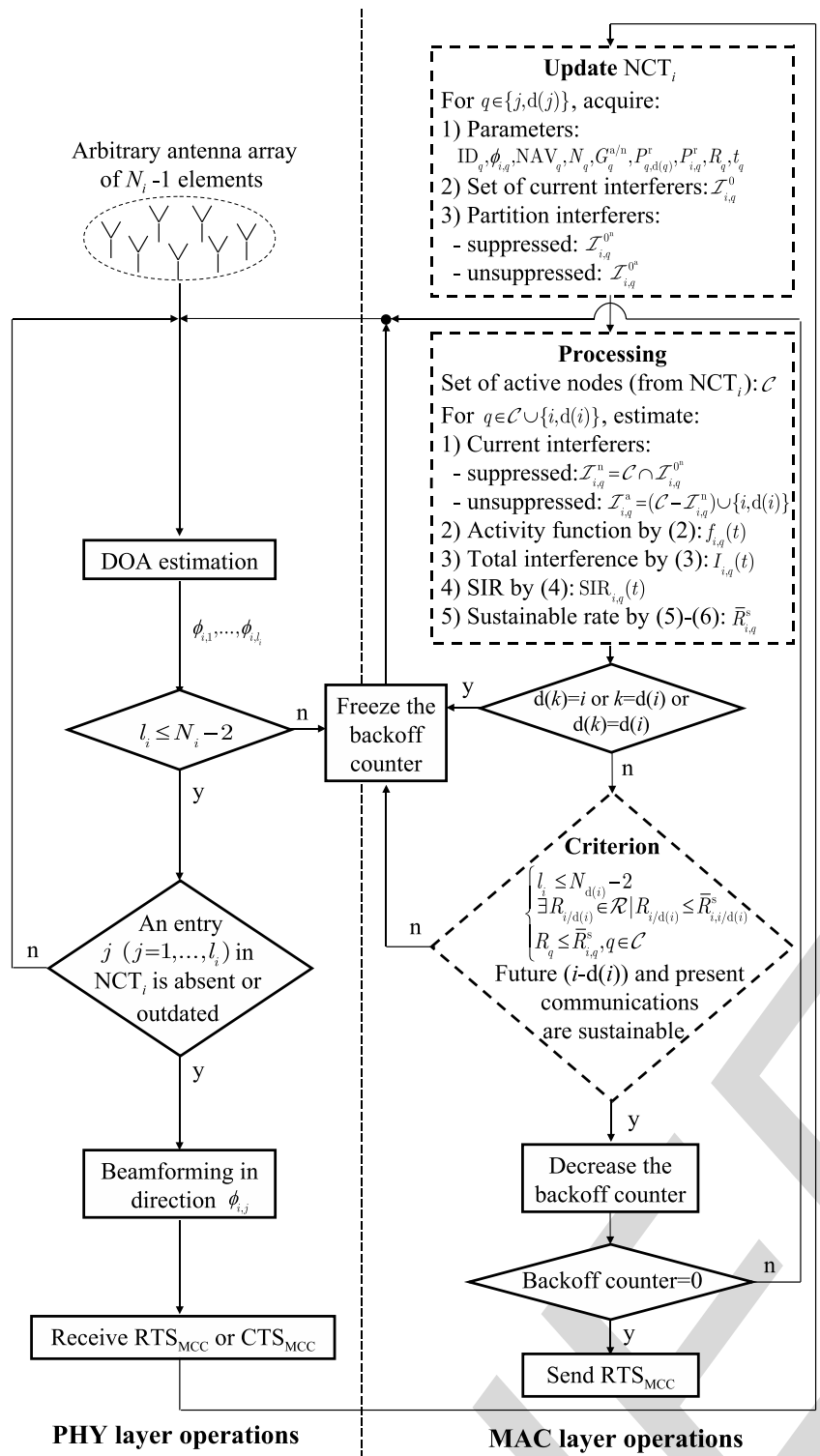

Fig. 3. SAMPC: sensing and backoff decrease rules in the MCC for node $i$.

for the nodes $p$ with which $j$ has already established a communication.

\subsection{Operations in the MCC: Multiple Communications}

Similarly to the TAMPC protocol, when a transmission on the MCC is sensed, the node $i$ estimates the current DOAs and checks in the $\mathrm{NCT}_{i}$ if one DOA corresponds to a NAV equal to zero, to detect whether a new communication $j$-d $(j)$ has started (Fig. 3). In this case, $i$ runs its beamforming algorithm to receive the $\mathrm{RTS}_{\mathrm{MCC}} / \mathrm{CTS}_{\mathrm{MCC}}$ packets, which, together with the IDs and the NAVs, now contain $N_{j / \mathrm{d}(j)}, G_{j / \mathrm{d}(j)}^{\mathrm{a} / \mathrm{n}}, P_{j, \mathrm{~d}(j)}^{\mathrm{r}}, P_{\mathrm{d}(j), j}^{\mathrm{r}}$, and the code rates $R_{\mathrm{d}(j)}$ and $R_{j}$ used for the corresponding DATA and ACK transmissions, respectively. These quantities are reinserted in the $\mathrm{RTS}_{\mathrm{MCC}}$ / CTS $_{\text {MCC }}$ packets to allow a node that has lost the first $j-\mathrm{d}(j)$ exchange in the CC to acquire the parameters of $j$ and $\mathrm{d}(j)$. After the $\mathrm{RTS}_{\mathrm{MCC}} / \mathrm{CTS}_{\mathrm{MCC}}$ reception and the estimations of the DOAs $\phi_{i, j / \mathrm{d}(j)}$ and of the received powers $P_{i, j / \mathrm{d}(j)}^{\mathrm{r}}, i$ first derives the instant of beginning of the corresponding DATA reception $t_{\mathrm{d}(j)}$ and that of the relative ACK reception $t_{j}=t_{\mathrm{d}(j)}+T_{\mathrm{D}}+$ SIFS, where $T_{\mathrm{D}}$ is the transmission time of the DATA frame and SIFS is the short interframe space. Secondly, for each of the two nodes $q \in\{j, \mathrm{~d}(j)\}, i$ stores the ordered set $\mathcal{I}_{i, q}^{0}=\left\{p \in \mathcal{N}^{\mathrm{n}} \mid \mathrm{NAV}_{p}>0 ; p \neq q, \mathrm{~d}(q) ; P_{q, 1}^{\mathrm{r}} \leq \cdots\right.$ $\left.\leq P_{q, p}^{\mathrm{r}} \leq \cdots \leq P_{q, l_{i}}^{\mathrm{r}}\right\}$ of the estimated $l_{i}$ active interferers of $q$ in the $q$-th entry of the $\mathrm{NCT}_{i}$. The set $\mathcal{I}_{i, q}^{0}$ is generated by $i$ taking $q$ as reference, to account for the impossibility of preserving the interference configuration in an asynchronous scenario [18]. Thus, if $i$ intends to perform a reliable SIR estimation for a node $q$, the initial interference configuration experienced by $q$ must be stored. Since adaptive arrays aim to maximize the SIR [39], $i$ can reasonably assume that, for a node $q$ with $N_{q}-1$ antennas $\left(N_{q}-2\right.$ degrees of freedom), the $N_{q}-3$ available nulls are used to suppress the strongest interferers in $\mathcal{I}_{i, q}^{0}$, while the other interferers are received with the average gain $G_{q}^{\mathrm{a}}$ [11]. Thus, $\mathcal{I}_{i, q}^{0}$ can be partitioned in two subsets: $\mathcal{I}_{i, q}^{0^{\mathrm{n}}}$ containing the $N_{q}-3$ suppressed interferers, and $\mathcal{I}_{i, q}^{0^{a}}$, containing the other ones.

The stored information can be used by a source $i$ to decide whether to decrease or not the backoff counter according to the SIR estimated not only for the pair $i-\mathrm{d}(i)$, but also for all pairs active on the MCC. To this aim, $i$ considers the set $\mathcal{C}$ containing the nodes currently involved in a communication on the MCC. For each $q \in \mathcal{C}, i$ evaluates the set $\mathcal{I}_{i, q}^{\mathrm{n}}=\mathcal{C} \cap \mathcal{I}_{i, q}^{0^{\mathrm{n}}}$, containing the still active interferers suppressed by $q$, and the set $\mathcal{I}_{i, q}^{\mathrm{a}}=\left(\mathcal{C}-\mathcal{I}_{i, q}^{\mathrm{n}}\right) \cup\{i, \mathrm{~d}(i)\}$, containing the still present not suppressed interferers of $q$ together with $i$ and $\mathrm{d}(i)$. For $q \in\{i, \mathrm{~d}(i)\}, i$ just partitions $\mathcal{C}$ into the subsets $\mathcal{I}_{i, q}^{\mathrm{n}}$, containing the $N_{q}-3$ suppressible interferers, and $\mathcal{I}_{i, q^{\prime}}^{\mathrm{a}}$ containing the not suppressible ones. Besides, for each $q \in \mathcal{C} \cup\{i, \mathrm{~d}(i)\}, i$ defines the indicator function:

$$
f_{i, q}(t)= \begin{cases}1 & t_{q} \leq t \leq t_{q}+T_{\mathrm{A}}, q \in \mathcal{S} \cup\{i\} \\ 1 & t_{q} \leq t \leq t_{q}+T_{\mathrm{D}}, q \in \mathcal{D} \cup\{\mathrm{d}(i)\} \\ 0 & \text { elsewhere }\end{cases}
$$

where $T_{\mathrm{A}}$ is the duration of the ACK reception, $\mathcal{S}(\subset \mathcal{C})$ is the subset of the active sources, and $\mathcal{D}(\subset \mathcal{C})$ is the subset of the active destinations. The function in (2) describes the activity of the node $q$ as a function of the time $t$. For $q \in \mathcal{C}, t_{q}$ is stored in the $q$ th entry of the $\mathrm{NCT}_{i}$, while, for $q \in\{i, \mathrm{~d}(i)\}, t_{q}$ is the current time. Moreover, by setting a counter for each $q \in \mathcal{N}^{\mathrm{n}}, i$ can estimate the probability of activity $\eta_{i, q}$ as the ratio between the number of packets sent by $q$ and the overall packets sensed on the MCC. Now, $i$ can estimate the time evolution of the interference experienced by each node $q \in \mathcal{C} \cup\{i, \mathrm{~d}(i)\}$ as:

$$
\begin{aligned}
\mathrm{I}_{i, q}(t)= & G_{q}^{\mathrm{n}} \sum_{p \in \mathcal{I}_{i, q}^{\mathrm{n}}} f_{i, p}(t) P_{q, p}^{\mathrm{r}}+G_{q}^{\mathrm{a}} \sum_{p \in \mathcal{I}_{i, q}^{\mathrm{a}}} f_{i, p}(t) P_{q, p}^{\mathrm{r}} \\
& +G_{q}^{\mathrm{a}} \sum_{p \in \mathcal{N}^{\mathrm{n}}-\{q\}} \eta_{i, p} P_{q, p}^{\mathrm{r}} .
\end{aligned}
$$

The first two terms in (3) are deterministic and account for the time evolution of the surely active interferers, while the third term estimates the probability that each non-legacy node becomes an interferer. Observe that the set $\mathcal{N}^{\mathrm{n}}-\{q\}$ 
in the third term includes also the currently active nodes, because a node that has completed its transmission may begin a further one. From (3), the SIR for the node $q$ can be derived as:

$$
\operatorname{SIR}_{i, q}(t)=\frac{1}{\mathrm{I}_{i, q}(t)} \cdot \begin{cases}P_{q, \mathrm{~d}(q)}^{\mathrm{r}} & q \in \mathcal{S} \cup\{i\} \\ P_{\mathrm{s}(q), q}^{\mathrm{r}} & q \in \mathcal{D} \cup\{\mathrm{d}(i)\},\end{cases}
$$

where $\mathrm{s}(q)$ is the source of the packet having $q$ as destination.

Once all SIRs are available, a reliable criterion for estimating the result of a communication using LDPC codes can be derived from the sustainable code rate [41]. This criterion considers the instantaneous SIR and, according to the modulation, provides a code rate function $R_{i, q}^{\mathrm{s}}(t)$, estimated using the sphere packing bound, whose average value is compared to the selected code rate $R_{q}$ [41]. For example, using the quadrature phase-shift keying (QPSK) modulation adopted by the 802.11 PHY layer [1], [7], a conservative value of $R_{i, q}^{\mathrm{s}}(t)$ is [13]:

$$
R_{i, q}^{\mathrm{S}}(t)=1-\log _{2}\left[1+e^{-\operatorname{SIR}_{i, q}(t) / 2}\right],
$$

from which one can obtain the sustainable code rate as:

$$
\bar{R}_{i, q}^{\mathrm{s}}=\frac{1}{T_{q}} \int_{0}^{T_{q}} R_{i, q}^{\mathrm{s}}(t) \mathrm{d} t,
$$

where $T_{q}=T_{\mathrm{D}}$ if $q$ is a destination, while $T_{q}=T_{\mathrm{A}}$ if $q$ is a source. A packet may be assumed successfully received if the adopted code rate $R_{q}$ satisfies $R_{q} \leq \bar{R}_{i, q}^{\mathrm{s}}$ [41]. Thus, defining as $\mathcal{R}$ the set of the selectable code rates, the three conditions:

$$
\left\{\begin{array}{l}
l_{i} \leq N_{\mathrm{d}(i)}-2 \\
\exists R_{i}, R_{\mathrm{d}(i)} \in \mathcal{R} \mid R_{i} \leq \bar{R}_{i, i}^{\mathrm{s}}, R_{\mathrm{d}(i)} \leq \bar{R}_{i, \mathrm{~d}(i)}^{\mathrm{s}}, \\
R_{q} \leq \bar{R}_{i, q}^{\mathrm{s}}, q \in \mathcal{C}
\end{array}\right.
$$

are used by $i$ as the criterion for decreasing its backoff counter. The first condition requires that $\mathrm{d}(i)$ can estimate $l_{i}$, thus avoiding deafness, while the others require that, if the communication $i-\mathrm{d}(i)$ becomes active, this communication and the currently active ones are successful. The SAMPC scheme considers the potential effect of the concurrent transmissions not only on the DATA receptions, but also on the ACK receptions, which are often neglected but can have a considerable impact on the result of the communication attempt. Hence, the SAMPC protocol adopts an advanced collision avoidance policy based on an accurate estimation of the instantaneous SIR and of the code behavior, in order to allow each node to evaluate the effect of the interference on its potential communication and on all the active ones, taking into account both the DATA and the ACK receptions.

Summarizing, the TAMPC and SAMPC schemes acquire information on the current medium occupation, thus matching the first condition stated in [18] for exploiting adaptive arrays. Additionally, the SAMPC protocol estimates the evolution of the interference, in order to compensate for the impossibility of preserving the interference configuration in a distributed scenario [18]. Differently from the 802.11n extension [7], which provides just a single high data rate communication at a time, the TAMPC and SAMPC schemes enable multiple simultaneous communications between different node pairs, without affecting the communications of the 802.11 legacy nodes, since the RTS/CTS packets in the CC are not modified, the More Data field is not read by the legacy nodes during non centralized operations, the DATA/ACK packets containing novel fields in the $\mathrm{CC}$ are not received by the legacy nodes that turn off their radios for the NAV duration, and all modifications in the MCC are transparent to the legacy nodes, whose activity is limited to the $\mathrm{CC}$. Besides, thanks to the priority guaranteed to the CC on the MCC, the legacy nodes do not suffer deafness.

\section{Computational Analysis}

Since the proposed protocols involve MAC/PHY procedures more sophisticated than the standard ones, an estimation of the number of required operations can be useful for clarifying some implementation aspects. To simplify the analysis, assume that just one floating point operation (flop), namely an addition, a multiplication, or a comparison, can be performed in a clock cycle. The modern digital signal processors (DSPs) capable to support the computational cost of the forthcoming 802.11 extensions are able to perform floating point operations at more than $2.8 \mathrm{Gflops} / \mathrm{s}$ [43], [44], [45], thus one may estimate, conservatively, that 1 flop requires $T_{\mathrm{f}} \cong 0.36 \mathrm{~ns}$.

The computational burden of the antenna processing algorithms represents the first element that must be taken into account. Two widely used methods are the multiple signal classification (MUSIC) algorithm, for the estimation of the DOAs, and the constrained least mean square (LMS) algorithm, for the synthesis of the array radiation pattern. One of the main reasons for the adoption of these techniques lies in the low number of operations with respect to other existing solutions [39]. For such reasons, the MUSIC and the LMS algorithms are adopted for the antenna processing operations of both the proposed protocols. In particular, considering an antenna system of $N_{i}-1$ elements and using $K$ samples to discretize the azimuth domain, the number of flops required by the MUSIC algorithm to estimate $l_{i}$ DOAs is $l_{i}\left(N_{i}-1\right)^{2}+\left(N_{i}-l_{i}-1\right) N_{i} K$ [46], while the number of flops required by the LMS algorithm to evaluate the array excitations in $K^{\prime}$ iterations can be reduced to $2 N_{i} K^{\prime}$ [47]. Therefore, the time required to complete the antenna processing operations is:

$$
t_{\mathrm{P}}=\left\{l_{i}\left(N_{i}-1\right)^{2}+N_{i}\left[\left(N_{i}-l_{i}-1\right) K+2 K^{\prime}\right]\right\} T_{\mathrm{f}} .
$$

While the previous estimation, involving just the PHY layer, is identical for both the TAMPC and SAMPC schemes, at the MAC layer the two access methods are characterized by different computational burdens. In particular, the TAMPC protocol requires just the evaluation of (1), which involves $l_{i}+2$ comparisons, leading to a computational time equal to:

$$
t_{\mathrm{M}}^{\mathrm{T}}=\left(l_{i}+2\right) T_{\mathrm{f}}
$$

The SAMPC protocol, instead, requires more calculations. For a fixed node $q$ and a fixed time slot, the first two terms in (3) require $2\left(l_{i}+1\right)$ multiplications and $l_{i}+1$ 
summations, while the third term implies just a single summation, since, being this term not dependent on the time, it does not involve real-time estimations and can be derived offline. The calculation of each sample of the SIR in (4) involves a division, which typically requires four flops. Thus, the evaluation of the SIR in a slot requires $2\left(l_{i}+1\right)+$ $\left(l_{i}+1\right)+1+4=3 l_{i}+8$ flops. To reduce the computational complexity due to the evaluation of (5) from the SIR, a quantization to $b$ bits can be used. Hence, adopting a bisection method to associate the calculated SIR to the corresponding value of the code rate function, the number of operations becomes $3 l_{i}+8+b$ for each node $q$ in a slot. Since this estimation must be performed for all slots covered by a packet, the number of flops required to estimate the code rate function of each node $q$ is $\left(3 l_{i}+8+b\right) \cdot T_{q_{\mathrm{n}}}$, where, denoting as $\lceil\cdot\rceil$ the ceiling function, $T_{q_{\mathrm{n}}}=\left\lceil T_{q} / \tau\right\rceil$ is the packet transmission time normalized to the slot time $\tau$. The evaluation in (6) of the mean value of the code rate function requires $T_{q_{\mathrm{n}}}$ summations and a division, while the comparison in (7) requires one flop. This leads to $\left(3 l_{i}+8+b\right) T_{q_{\mathrm{n}}}+\left(T_{q_{\mathrm{n}}}+4\right)+$ $1=\left(3 l_{i}+9+b\right) T_{q_{\mathrm{n}}}+5$ flops for estimating and comparing the sustainable code rate of each node $q$ with the selected code rate. Considering the $l_{i}$ active sources, the sensing one, and their destinations, one can infer that $2 l_{i}+2$ nodes are involved $\left(l_{i}+1\right.$ concerning a DATA packet and $l_{i}+1$ concerning an ACK packet). Thus, the computational time required by the SAMPC protocol for estimating the result of all $2 l_{i}+2$ receptions and that of the comparison $l_{i} \leq N_{\mathrm{d}(i)}-$ 2 in (7) is:

$$
t_{\mathrm{M}}^{\mathrm{S}}=\left\{\left(l_{i}+1\right)\left[\left(3 l_{i}+9+b\right)\left(\left\lceil\frac{T_{\mathrm{D}}}{\tau}\right\rceil+\left\lceil\frac{T_{\mathrm{A}}}{\tau}\right\rceil\right)+10\right]+1\right\} T_{\mathrm{f}} .
$$

\section{Results}

The proposed protocols have been implemented in a $\mathrm{C}++$ simulation platform developed using the IT++ libraries for signal processing and communications [50]. The selected algorithms and parameters are reported in Table 1, and a geometrically distributed payload is adopted [34]. For each simulation, 60 seconds of network activity are monitored. At the beginning of each simulation, all transmission queues are empty and none of the nodes has previous information concerning the characteristics of the other nodes or of the topology. To simplify the comparison between the two schemes, a fixed code rate $R_{i}=2 / 3$ is assumed for the ACK packets in all the simulations, while the value of the code rate $R_{\mathrm{d}(i)}$ for the DATA packets, which is still maintained fixed, will be specified for each examined scenario. The LDPC codes are adopted also for the TAMPC protocol, where, however, they simply replace the standard convolutional codes, but are not involved in the backoff counter process as in the SAMPC protocol. Besides, the threshold $L_{\mathrm{t}_{i}}$ used by the generic non-legacy node $i$ for the TAMPC scheme is assumed to be equal to $N_{i}-2$ [34]. The QPSK modulation has been selected because, among the higher-order modulations, it does not force to adopt very low source-destination distances to operate in a lowrank channel with the typical path-loss attenuations, and, furthermore, it represents an interesting application of the sustainable code rate concept thanks to the possibility
TABLE 1

Adopted Parameters and Algorithms

\begin{tabular}{ll}
\hline & Common parameters \\
\hline Slot time & $20 \mu \mathrm{s}$ \\
Distributed InterFrame Space & $50 \mu \mathrm{s}$ \\
Short InterFrame Space & $10 \mu \mathrm{s}$ \\
Maximum backoff stage & 4 \\
Retry limit & 4 \\
RTS & 160 bits \\
CTS & 112 bits \\
DATA header & 240 bits \\
Average payload & 6,960 bits \\
ACK & 112 bits \\
Data rate & 12 Mbits/s \\
Control rate & 2 Mbits /s \\
Modulation & QPSK \\
Preamble & 128 bits \\
Transmission power & $20 \mathrm{dBm}$ \\
Noise power & $-100.8 \mathrm{dBm}$ [48] \\
Path-loss exponent $\alpha$ & 3 \\
SIR reception threshold & 2 dB [49] \\
Commmunication range & $90 \mathrm{~m}$ \\
Interference range & $90 \mathrm{~m}$ \\
Antenna array geometry & Uniform circular array \\
DOA estimation algorithm & MUSIC [39] \\
Beamforming algorithm & Constrained LMS [39] \\
\hline &
\end{tabular}

\begin{tabular}{ll}
\hline & TAMPC parameters \\
\hline$L_{\mathrm{t}_{i / \mathrm{d}(i)}}$ & 4 bits \\
RTS $_{\mathrm{MCC}}$ & RTS $+L_{\mathrm{t}_{i}}+$ preamble \\
$\mathrm{CTS}_{\mathrm{MCC}}$ & $\mathrm{CTS}+L_{\mathrm{t}_{\mathrm{d}(i)}}+$ preamble \\
\hline & SAMPC parameters \\
\hline$N_{i / \mathrm{d}(i)}$ & 4 bits \\
$G_{i / \mathrm{d}(i)}^{\mathrm{n} / \mathrm{a}}$ & 8bits \\
$R_{i / \mathrm{d}(i)}$ & 2 bits \\
$\mathrm{RTS}_{\mathrm{MCC}}$ & RTS $+N_{i}+G_{i}^{\mathrm{n}}+G_{i}^{\mathrm{a}}+R_{i}+$ preamble \\
$\mathrm{CTS}_{\mathrm{MCC}}$ & $\mathrm{CTS}+N_{\mathrm{d}(i)}+G_{\mathrm{d}(i)}^{\mathrm{n}}+G_{\mathrm{d}(i)}^{\mathrm{a}}+R_{\mathrm{d}(i)}+$ preamble \\
\hline
\end{tabular}

to evaluate $R_{i, q}^{\mathrm{s}}(t)$ in closed form by (5). To this purpose, it is worth noticing that, for the further higher-order modulations, the code rate function may involve more complex expressions, whose calculation, however, do not affect the computational cost, since, as discussed in the previous section, the association between the SIR and the corresponding value of the code rate function is based on a quantization process.

The adopted performance figures are the throughput and the Jain's fairness index. The throughput, denoting the ratio between the number of slots covered by successful receptions and the overall number of simulated slots at the net of the adopted code rate, directly provides the number of simultaneous communications that can be hosted in a slot by the TAMPC and SAMPC schemes. The presented results are subdivided in two main parts. The first part simulates the proposed schemes in heterogeneous scenarios involving both legacy and non-legacy nodes equipped with different antenna systems in asymmetric network topologies (Sections 8.1, 8.2, 8.3, 8.4, and 8.5). The second part discusses the protocols' behavior with respect to the analysis of [13] in a symmetric topology, and compares the presented solution with the 802.11n extension in a multipath-fading environment (Sections 8.6 and 8.7). 


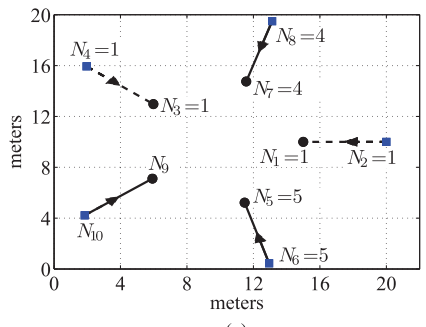

(a)

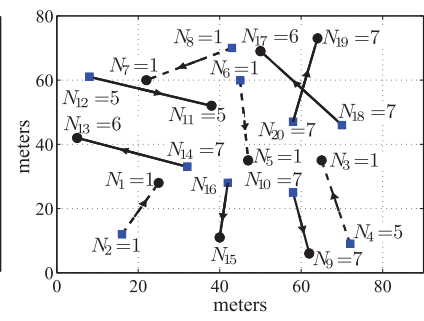

(b)
Fig. 4. Heterogeneous scenarios: a) ring topology, b) random topology. - - - Legacy communication —-Non-legacy communication.

\subsection{Basic Topologies}

The first set of results is obtained considering a minimum contention window equal to 32 and a code rate $R_{i}=8 / 9$ in the presence of a Poisson packet arrival process. The adopted topologies with the corresponding $N_{i}$ values are shown in Fig. 4a, reporting a ring topology with two legacy and three non-legacy pairs, and in Fig. 4b, reporting a random topology with four legacy and six non-legacy pairs. In the first topology the nodes are placed on two concentric rings, where the sources lie in the outer ring, having a radius equal to $20 \mathrm{~m}$, and the destinations lie in the inner ring, having a radius equal to $10 \mathrm{~m}$. In the second topology the communicating pairs are randomly placed within a circle of radius equal to $45 \mathrm{~m}$. In both cases each node lies within the communication range of the others. The number of antennas of the pair 9-10 in the ring topology and that of the pair 15-16 in the random topology will be set each to two different values, thus allowing the development of two different scenarios for each topology. These values will be selected to better put into evidence the mode of operation of the two presented schemes.

The aggregate throughput, given by the sum of the throughput in the CC and in the MCC, is shown in Fig. 5a, which refers to the topology in Fig. 4 a considering the cases $N_{9,10}=4$ and $N_{9,10}=3$, and in Fig. $5 \mathrm{~b}$, which refers to the topology in Fig. $4 \mathrm{~b}$ considering the cases $N_{15,16}=7$ and $N_{15,16}=3$. To provide a reference, the figures also report the throughput obtained using the 802.11 super-g technology [51], which is an $802.11 \mathrm{~g}$-based commercial extension that bonds two adjacent (non-overlapping) $20 \mathrm{MHz}$ channels to obtain a spectrum of $40 \mathrm{MHz}$, thus doubling the data rate

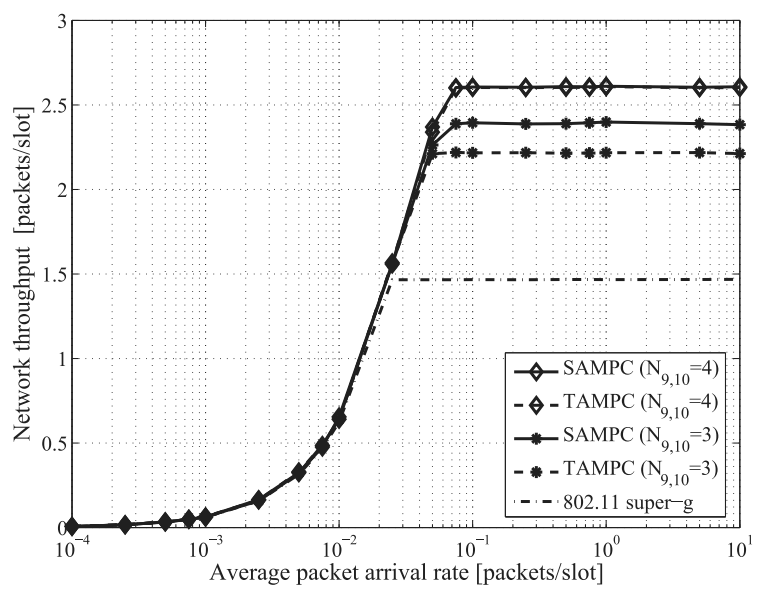

(a) for the single allowed communication. Therefore, since the three solutions (TAMPC, SAMPC, and 802.11 super-g) use the same amount of spectrum resources, one can obtain a fair comparison between an existing technology and the here proposed designs. Concerning the interpretation of the results in Fig. 4, it is useful to recall that the adopted throughput definition allows one to immediately infer the number of simultaneous communications, each characterized by a data rate of $12 \mathrm{Mbits} / \mathrm{s}$, effectively provided by the TAMPC and SAMPC schemes. For example, considering the SAMPC protocol for the case $N_{9,10}=4$ in Fig. $4 \mathrm{a}$, a throughput approximately equal to 2.61 packets/slot implies that 2.61 simultaneous communications at $12 \mathrm{Mbits} / \mathrm{s}$ have been simultaneously active. However, this direct relationship throughput-simultaneous communications does not hold for the curves referred to the 802.11 super-g extension, since in this case a unique communication is active, but with a data rate doubled with respect to that used by the TAMPC and SAMPC schemes. This is the reason for the presence of throughput values larger than one also for the 802.11 super-g extension, which are referred to a single communication at $24 \mathrm{Mbits} / \mathrm{s}$.

The curves show that both the TAMPC and SAMPC protocols provide a higher performance with respect to the super-g extension, allowing the coexistence of a number of simultaneous communications ranging from two to three for all the considered scenarios. With reference to Fig. 5a, a direct comparison between the two developed schemes reveals that, when $N_{9,10}=4$, their throughput is substantially identical, while, when $N_{9,10}=3$, the SAMPC scheme outperforms the TAMPC one. These two values of $N_{9,10}$ have been specifically selected to clearly outline how the two protocols behave. To this purpose, consider also the single-node saturation throughput in Table 2. For $N_{9,10}=4$, the minimum threshold among all nodes operating on the MCC is $L_{\mathrm{t}_{i}}^{\mathrm{m}}=4-2=2$, and, as described in Section 5.2, using the TAMPC protocol the backoff counter is decreased when the number of estimated active sources $l_{i}$ is lower or equal to $L_{\mathrm{t}_{i}}^{\mathrm{m}}$. This implies that when two communications are active, a further one can be established and hence three transmissions may be simultaneously performed on the MCC. Considering also the CC, a total of four concurrent transmissions may be sustained by the

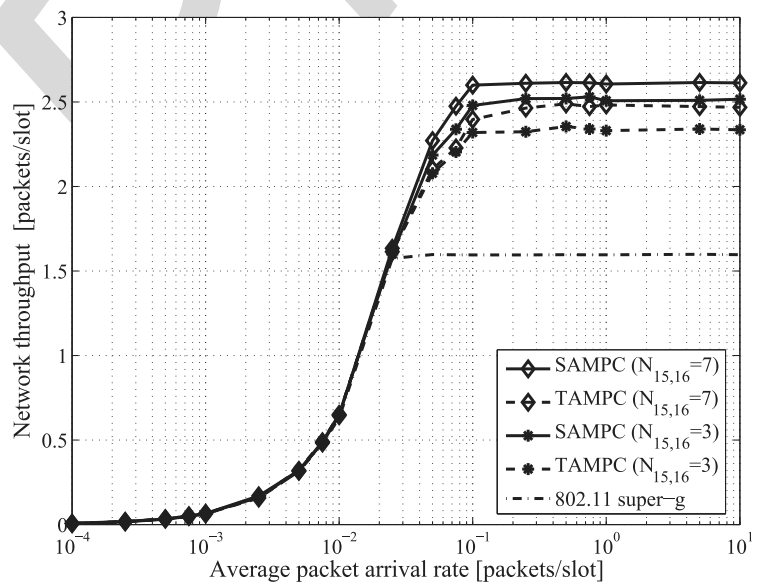

(b)

Fig. 5. Network throughput as a function of the average arrival rate: a) topology in Fig. 4a, b) topology in Fig. 4b. 
TABLE 2

Single-Pair Throughput (Packets/Slot) for Topologies in Fig. 4

\begin{tabular}{|c|c|c|c|c|}
\hline \multicolumn{5}{|c|}{ Topology in Fig. 4 a $(1 \div 4$ legacy, $5 \div 10$ non-legacy) } \\
\hline & \multicolumn{2}{|c|}{$N_{9,10}=4$} & \multicolumn{2}{|c|}{$N_{9,10}=3$} \\
\hline Pair & TAMPC & SAMPC & TAMPC & SAMPC \\
\hline$\overline{1-2}$ & 0.38 & 0.38 & 0.38 & 0.38 \\
\hline $3-4$ & 0.38 & 0.38 & 0.38 & 0.38 \\
\hline $5-6$ & 0.62 & 0.62 & 0.50 & 0.62 \\
\hline $7-8$ & 0.62 & 0.62 & 0.49 & 0.62 \\
\hline $9-10$ & 0.60 & 0.61 & 0.47 & 0.39 \\
\hline Fairness (CC) & 1.00 & 1.00 & 1.00 & 1.00 \\
\hline Fairness (MCC) & 1.00 & 1.00 & 1.00 & 0.96 \\
\hline \multicolumn{5}{|c|}{ Topology in Fig. 4b $(1 \div 8$ legacy, $9 \div 20$ non-legacy $)$} \\
\hline & \multicolumn{2}{|c|}{$N_{15,16}=7$} & \multicolumn{2}{|c|}{$N_{15,16}=3$} \\
\hline Pair & TAMPC & SAMPC & TAMPC & SAMPC \\
\hline $1-2$ & 0.22 & 0.22 & 0.22 & 0.22 \\
\hline $3-4$ & 0.20 & 0.20 & 0.20 & 0.20 \\
\hline $5-6$ & 0.19 & 0.19 & 0.19 & 0.19 \\
\hline $7-8$ & 0.21 & 0.21 & 0.21 & 0.21 \\
\hline $9-10$ & 0.60 & 0.54 & 0.56 & 0.58 \\
\hline $11-12$ & 0.03 & 0.08 & 0.02 & 0.05 \\
\hline $13-14$ & 0.03 & 0.14 & 0.37 & 0.40 \\
\hline $15-16$ & 0.60 & 0.46 & 0.09 & 0.05 \\
\hline $17-18$ & 0.15 & 0.34 & 0.07 & 0.35 \\
\hline $19-20$ & 0.23 & 0.24 & 0.42 & 0.27 \\
\hline Fairness (CC) & 1.00 & 1.00 & 1.00 & 1.00 \\
\hline Fairness (MCC) & 0.56 & 0.76 & 0.60 & 0.69 \\
\hline
\end{tabular}

network. Observe that the adoption of a large minimum contention window, selected equal to 32, and the presence of the channel encoder, determine throughput values lower than four, as it can be shown in Fig. 5 a for $N_{9,10}=4$. However, regardless of these aspects, in the case $N_{9,10}=4$ the proposed protocols guarantee the same throughput and a fair access to the network nodes. By contrast, in the case $N_{9,10}=3$, the SAMPC protocol provides a higher throughput, while the TAMPC scheme maintains a better fairness. This result can be explained analyzing the access of the nodes operating on the MCC, namely the pairs 5-6, 7-8, and 9-10, for the two developed schemes. For $N_{9,10}=3$, the thresholds become $L_{\mathrm{t}_{5 \div 8}}=2$ and $L_{\mathrm{t}_{9 \div 10}}=1$. Adopting both protocols, when the pairs 5-6 and 7-8 (having four antennas) are active, the source 10 (having just three antennas) freezes its backoff counter because it cannot estimate more than one active transmitter. A different behavior appears for the two schemes in another configuration of transmitters. Adopting the TAMPC protocol, when one of the two pairs with more than three antennas (5-6 or 7-8) and the unique pair with three antennas (9-10) are active, the source of the other pair with more than three antennas ( 8 or 6 , respectively) freezes its backoff counter to protect the communication of the pair 9-10, according to the criterion on the minimum threshold in (1). This implies a fair reduction of the single-node throughput. Conversely, adopting the SAMPC scheme in the same configuration of transmitters, the source 8 (or 6) does not freeze its backoff counter, since it estimates that the communication of the pair 9-10 remains successful in the presence of the other two communications. Hence, the pairs with three antennas acquire more resources, leading to a higher throughput at the
TABLE 3

Impact of DOA and RSSI Estimation Errors on the Throughput (Packets/Slot) for Topology in Fig. 4b with $N_{15,16}=7$

\begin{tabular}{|c|c|c|c|c|c|c|}
\hline$\sigma_{\mathrm{RSSI}}[\mathrm{dB}]$ & $-\infty$ & -10 & -5 & 0 & 5 & 10 \\
\hline \multicolumn{7}{|l|}{$\sigma_{\mathrm{DOA}}[\mathrm{deg}]$} \\
\hline$\overline{0}$ & 1.81 & 1.81 & 1.81 & 1.03 & 0.88 & 0.86 \\
\hline 5 & 1.81 & 1.81 & 1.81 & 1.01 & 0.88 & 0.86 \\
\hline 10 & 1.59 & 1.59 & 1.59 & 0.99 & 0.86 & 0.85 \\
\hline 20 & 1.11 & 1.11 & 1.11 & 0.89 & 0.82 & 0.81 \\
\hline
\end{tabular}

expense of a slight fairness reduction. The same considerations can be formulated for the second topology when moving from $N_{15,16}=7$ to $N_{15,16}=3$, which confirm the capability of the SAMPC scheme to more reliably estimate the channel conditions that allow a node to attempt a transmission in the MCC.

\subsection{Impact of Estimation Errors}

In both proposed protocols each node tries to protect not only its own communication but also all the other active ones. The TAMPC protocol reaches this objective by accounting for the load threshold sustainable by the active node with the less powerful antenna system, without considering the network topology. The SAMPC protocol also adopts a protection mechanism, but, being based on a SIR estimation, this mechanism is able to account for a larger number of network elements, including not only the number of antennas, but also the antenna gains and the relative positions between the nodes. The values in Table 2 confirm that the adoption of this more reliable process of estimation of the network conditions provides considerable benefits to the global performance of the network itself. However, since the estimation process adopted by the SAMPC scheme relies on both the received power and the DOA, which are in turn two estimated quantities, it may be interesting to explore how a Gaussian error with null mean applied to the RSSI and to the DOA influences the throughput of the MCC. The values reported in Table 3 for the topology in Fig. $4 \mathrm{~b}$ with $N_{15,16}=7$ are obtained for different values of the standard deviation of the error on the RSSI $\sigma_{\mathrm{RSSI}}$ and of that on the DOA $\sigma_{\text {DOA }}$. One can observe that, until $\sigma_{\mathrm{RSSI}}$ is lower than $-5 \mathrm{~dB}$ (31 percent) and $\sigma_{\mathrm{DOA}}$ lies below 5 degrees, no performance degradations can be noticed with respect to the case in which errors are not present $\left(\sigma_{\mathrm{RSSI}}=-\infty, \sigma_{\mathrm{DOA}}=0\right)$. Hence, the SAMPC scheme is robust against RSSI and DOA estimation errors, which begin to have a relevant impact on the throughput when $\sigma_{\mathrm{RSSI}}$ becomes close to $0 \mathrm{~dB}$ (one order of magnitude) and/or $\sigma_{\mathrm{DOA}}$ approaches 10 degrees, values that are much worse than those typically offered by a wireless card and by the MUSIC algorithm. This confirms the usefulness of the SAMPC scheme for random topologies, which represent realistic scenarios for the commonly deployed wireless networks.

\subsection{Impact of the Number of Nodes}

To further investigate the performance of the two schemes in random topologies with a larger number of nodes, a third set of simulations is carried out by considering 50 random 


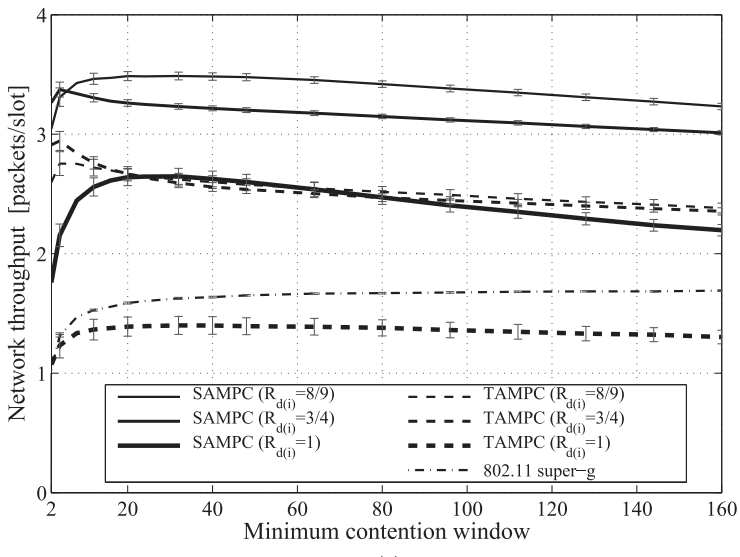

(a)

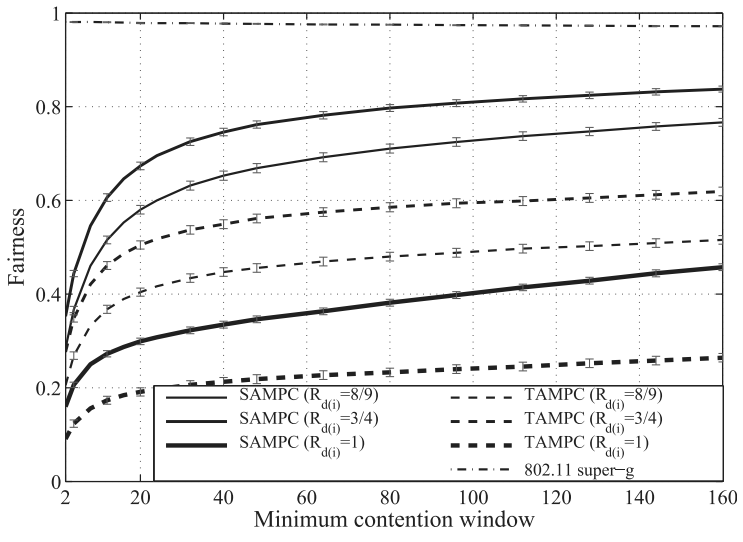

(b)

Fig. 6. Protocols' performance for $n=100$ nodes and 50 randomly generated topologies: a) network throughput, b) fairness.

topologies, each having $n$ non-legacy nodes with $N_{i}=6$, corresponding to $n / 2$ pairs able to operate on the MCC. Each random topology is generated by first displacing, according to a uniform distribution, the $n / 2$ sources on a circle of radius equal to $27 \mathrm{~m}$. Subsequently, each destination is displaced on a circle of radius equal to $18 \mathrm{~m}$ centered in the corresponding source, still according to a uniform distribution but referred to this second circle. Observe that, while the scenarios in Fig. 4 were heterogeneous both in terms of nodes and topologies, in this case the heterogeneity is just due to the asymmetry of the spatial distribution of identical nodes. Figs. $6 \mathrm{a}$ and $6 \mathrm{~b}$ report the average network saturation throughput and the fairness, respectively, as a function of the minimum contention window for $n=100$ nodes and different code rates, while Table 4 reports the same quantities for $R_{\mathrm{d}(i)}=3 / 4$ and a minimum contention window equal to 16 considering different values of $n$. The size of the confidence intervals in Fig. 6 are identified by the grey vertical bars. These results confirm the higher performance of the SAMPC protocol with respect to the TAMPC one for a given code rate. In particular, for the TAMPC protocol, the rates $3 / 4$ and $8 / 9$ lead to a similar throughput but the rate $3 / 4$ provides a higher fairness, while, for the SAMPC proto$\mathrm{col}$, the rate $8 / 9$ leads to a higher throughput, but not to a higher fairness, which is higher when adopting the rate $3 / 4$. However, a direct comparison between the uncoded cases $\left(R_{\mathrm{d}(i)}=1\right)$ and the coded ones reveals, on the one hand, that a certain channel coding is necessary to maintain an acceptable performance, but, on the other hand, that the redundancy can be kept low, since even a high code rate, such as $8 / 9$, can provide a satisfactory throughput, close to the one achievable using the lower rate of $3 / 4$. These results show that the capability of an antenna system alone does not

TABLE 4

Protocols' Performance for 50 Randomly Generated

Topologies and Different Values of $n$ Using $R_{\mathrm{d}(i)}=3 / 4$ and a Minimum Contention Window Equal to 16

\begin{tabular}{lccccccccc}
\hline Protocol & \multicolumn{3}{c}{ TAMPC } & \multicolumn{3}{c}{ SAMPC } & \multicolumn{3}{c}{802.11 super-g } \\
\hline$n$ & 20 & 50 & 100 & 20 & 50 & 100 & 20 & 50 & 100 \\
Throughput & 2.63 & 2.66 & 2.72 & 3.04 & 3.22 & 3.29 & 1.68 & 1.63 & 1.56 \\
[packets/slot] & & & & & & & & & \\
Fairness & 0.67 & 0.55 & 0.49 & 0.81 & 0.73 & 0.65 & 1.00 & 0.99 & 0.98 \\
\hline
\end{tabular}

represent an absolute element for the single-node performance, which is also influenced by its position within the network. Therefore, the use of a rigid threshold on the number of communications for establishing the result of a reception in an MPC scenario might sometimes lead to an under utilization of the network resources.

\subsection{Computational Burden and Energy Considerations}

An estimation of the computational burden of each node is now provided for the three investigated sets of scenarios: the topology in Fig. 4a, the topology in Fig. 4b, and the 50 random topologies with $n$ nodes. This estimation can be performed using (8)-(10) for $T_{\mathrm{f}} \cong 0.36 \mathrm{~ns}$ and considering, for each set of scenarios, the node with the highest number of antennas, $N_{\max }$, in the presence of the maximum number of active sources $l_{\max }=N_{\max }-2$ that it can detect on the MCC [34]. The time required by the antenna processing operations at the PHY layer can be evaluated assuming the adoption of $K=360$ samples in the azimuth domain for estimating the DOAs and $K^{\prime}=512$ iterations for synthesizing the radiation pattern using the constrained LMS algorithm. Thus, from (8), one obtains $t_{\mathrm{P}} \cong 2.5 \mu \mathrm{s}$ for the scenarios in Fig. $4 \mathrm{a}$ where $N_{\max }=5, t_{\mathrm{P}} \cong 3.5 \mu$ s for the scenarios in Fig. $4 \mathrm{~b}$ where $N_{\max }=7$, and $t_{\mathrm{P}} \cong 3.0 \mu \mathrm{s}$ for the 50 random topologies where $N_{\max }=6$. Since the preamble is transmitted at the control rate, its transmission requires $128 /\left(2 \cdot 10^{6}\right) \cong 64.0 \mu \mathrm{s}$ (Table 1 ), thus in both cases $t_{\mathrm{P}}<64.0 \mu \mathrm{s}$ and hence the capabilities of the commercially available DSPs for last generation standards are sufficient to sustain the computational requirements of the antenna processing. Using the same data, the time required by the MAC layer operations when the TAMPC protocol is used can be directly calculated from (9), which provides $t_{\mathrm{M}}^{\mathrm{T}} \cong 1.8$ ns for the scenarios in Fig. $4 \mathrm{a}, t_{\mathrm{M}}^{\mathrm{T}} \cong 2.5$ ns for the scenarios in Fig. $4 \mathrm{~b}$, and $t_{\mathrm{M}}^{\mathrm{T}} \cong 2.1 \mathrm{~ns}$ for the 50 random topologies. Instead, assuming a quantization with $b=8$ bits for the SIR and using (10) with the values in Table 1, the average computational time required by the SAMPC protocol is $t_{\mathrm{M}}^{\mathrm{S}} \cong 0.9 \mu \mathrm{s}$ for the scenarios in Fig. $4 \mathrm{a}, t_{\mathrm{M}}^{\mathrm{S}} \cong 1.9 \mu \mathrm{s}$ for the scenarios in Fig. $4 \mathrm{~b}$, and $t_{\mathrm{M}}^{\mathrm{S}} \cong 1.4 \mu \mathrm{s}$ for the 50 random topologies. As expected, the SAMPC protocol, performing more operations, requires a higher computational time than 
that of the TAMPC scheme. Despite this, $t_{\mathrm{M}}^{\mathrm{S}}$ is not prohibitive, since it remains in the order of few microseconds. Furthermore, it may be noticed that the presented computational analysis has been carried out adopting conservative assumptions for the hardware capabilities, since some DSPs supporting floating point operations are able to provide values of $T_{\mathrm{f}}$ lower than 0.36 ns [43], [44], [45]. This latter aspect can be considered even more true as the number of antennas of a router increases, since one can expect that more advanced devices are equipped with more powerful hardware.

These results suggest that the TAMPC scheme, being characterized by a lower computational burden and hence by a lower energy consumption, may be more suitable for pure ad-hoc scenarios, where a fixed feeding network is absent, but acceptable traffic levels must be sustained. Conversely, the SAMPC protocol, which requires more calculations and hence more energy, but achieves a higher throughput, may be more suitable for the backbone of a wireless mesh network, which has typically to provide all the contents supported by Internet connectivity, but can often rely on a fixed feeding network. Concerning these two different domains of application for the two proposed schemes, it may be useful to observe that the increase of the energy consumption due the PHY and MAC layers is of the same order of magnitude for the SAMPC protocol, while, for the TAMPC protocol, almost all consumption's increase is due to the PHY layer. In fact, considering the consumed energy as directly proportional to the number of operations and using (8)-(10), one can derive $t_{\mathrm{P}} / T_{\mathrm{f}}=8,404, t_{\mathrm{M}}^{\mathrm{T}} / T_{\mathrm{f}}=6$, and $t_{\mathrm{M}}^{\mathrm{S}} / T_{\mathrm{f}}=3,868$, corresponding to the number of operations of the common antenna processing procedures at $\mathrm{PHY}$ layer, of the TAMPC MAC layer, and of the SAMPC MAC layer, respectively. One may further observe from (8) that the main impact on the energy consumption due to the antenna processing is due to the number of samples $K$ used to discretize the azimuth domain, which in the presented simulations has been selected equal to 360 , thus considering a thick sampling. However, the results in Table 3 show that the adopted approach is characterized by a certain robustness against the DOA estimation errors, and hence lower $K$ values may be selected for ad-hoc networks when strong constraints on the energy consumption must be imposed.

\subsection{Impact of Preemptive Priority}

As explained in Section 4, a preemptive priority is guaranteed to the legacy sources when one of them sends an RTS to a non-legacy destination already involved in a communication on the MCC, so as to avoid deafness. To test the impact of this choice, the SAMPC scheme is applied to a network with four legacy nodes (two legacy, with $N_{1}=N_{2}=1$, and two non-legacy, with $N_{3}=N_{4}=5$ ) lying on a circumference of radius equal to $10 \mathrm{~m}$. Differently from the previous scenarios, where the traffic flows were fixed for the entire simulation, in this case, for each generated packet, each source randomly selects the destination. Therefore, in this scenario the pairs are not static, but can dynamically change during the simulation. Observe that the information concerning the characteristics of two communicating pairs are contained in the RTS/CTS (or $\mathrm{RTS}_{\mathrm{MCC}} / \mathrm{CTS}_{\mathrm{MCC}}$ ) packets. Hence, the composition of the communicating pairs is
TABLE 5

Performance on Each Link between Source $i$ and Destination $j$ for a Network with Randomly Selected Traffic Flows Involving Two Legacy $(i, j=1,2)$ and Two Non-Legacy $(i, j=3,4)$ Nodes

\begin{tabular}{|c|c|c|c|c|c|c|c|c|c|}
\hline & \multirow[b]{2}{*}{$i^{j}$} & \multicolumn{4}{|c|}{ No priority } & \multicolumn{4}{|c|}{ Preemptive priority to $\mathrm{CC}$} \\
\hline & & 1 & 2 & 3 & 4 & 1 & 2 & 3 & 4 \\
\hline Throughput & 1 & - & 0.06 & 0.06 & 0.06 & - & 0.09 & 0.09 & 0.09 \\
\hline \multirow[t]{3}{*}{ [packets/slot] } & 2 & 0.06 & - & 0.06 & 0.06 & 0.09 & - & 0.09 & 0.09 \\
\hline & 3 & 0.12 & 0.12 & - & 0.12 & 0.09 & 0.09 & - & 0.09 \\
\hline & 4 & 0.12 & 0.12 & 0.12 & - & 0.09 & 0.09 & 0.09 & - \\
\hline Drop & 1 & - & 0.00 & 2.66 & 2.10 & - & 0.00 & 0.00 & 0.00 \\
\hline probability & 2 & 0.00 & - & 2.50 & 2.57 & 0.00 & - & 0.00 & 0.00 \\
\hline due to & 3 & 0.00 & 0.00 & - & 0.00 & 0.00 & 0.00 & - & 0.00 \\
\hline deafness [\%] & 4 & 0.00 & 0.00 & 0.00 & - & 0.00 & 0.00 & 0.00 & - \\
\hline
\end{tabular}

continuously updated. In the case that the pairs are fixed, this information is redundant (except for the first packet exchange), while it becomes fundamental in the case of changing source-destination pairs. However, it is always present during the operations required by the two proposed protocols, thus the update is anyway guaranteed. In the simulated scenario a minimum contention window equal to 4 is used, in order to force situations in which a non-legacy node has to manage concurrent requests on both the CC and the MCC. Table 5 presents, for each link between each source $i$ and each destination $j$, the throughput and the drop probability referred to lost packets in which at least a retransmission was due to deafness. The results show that, not only the preemptive priority removes the losses due to deafness, which were in the order of 2-3 percent, but also improves the fairness.

\subsection{Comparison with Theory}

This section discusses the performance of the TAMPC protocol with respect to the analysis of [13], [15]. Since this analysis models a threshold-based access in a homogeneous scenario, a meaningful comparison with the theory can be obtained using the TAMPC scheme (with the settings in Table 1) in a symmetric topology. To this aim, $n=20$ identical non-legacy nodes are placed on two concentric rings, where the sources lie in the outer ring, having a radius of $20 \mathrm{~m}$, and the destinations lie in the inner ring, having a radius of $10 \mathrm{~m}$. Each node has $N_{i}=6(i \in \mathcal{N})$ antennas and selects a code rate $R_{i}=3 / 4(i \in \mathcal{N})$. Analysis and simulations are carried out according to the conditions adopted in [13], [15], with the purpose of validating the numerical results in that conditions.

Fig. 7 shows the network saturation throughput and the average successful packet delay as a function of the minimum contention window for the TAMPC protocol and the analysis of [13], [15]. The fairness results are not reported since they are close to unity in all cases. The analysis of [13], [15] and the TAMPC scheme are compared for the two thresholds $L_{\mathrm{t}_{i}}=L_{\mathrm{t}}=3(i \in \mathcal{N})$ and $L_{\mathrm{t}_{i}}=L_{\mathrm{t}}=5(i \in \mathcal{N})$. One can immediately observe that the analytic and the numerical results are very close for a large part of the considered range of minimum contention windows. Precisely, the theoretical and simulated throughput curves almost overlap when the minimum contention window is high, while differences appear when it becomes low, mainly for the case $L_{\mathrm{t}}=5$. These differences are due to the fact that the 


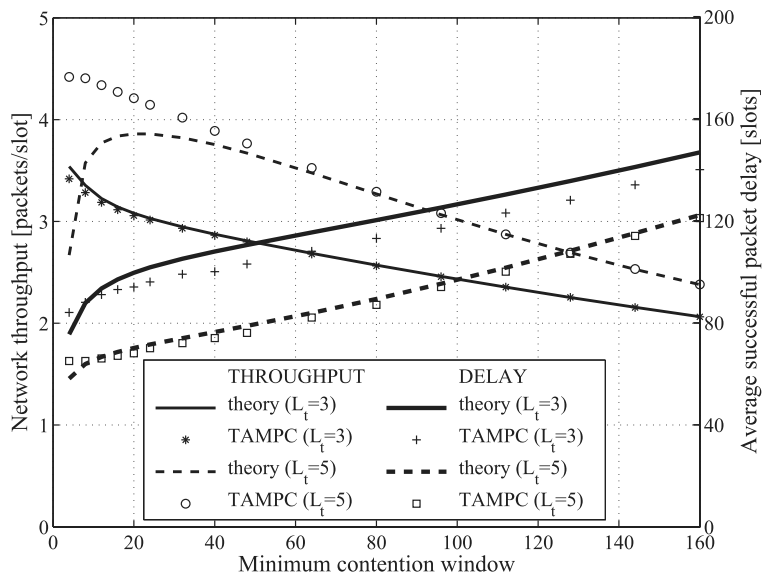

Fig. 7. Saturation throughput and average successful packet delay as a function of the minimum contention window.

studied scenario, even if symmetric and composed by identical nodes, is not perfectly homogeneous. In fact, in the simulation, the result of a reception does not depend only on the number of concurrent transmissions, but also on which transmitters are active. Hence, different configurations of the same number of active sources can lead in some cases to a success and in others to a failure. The analysis, instead, assuming a homogeneous scenario, considers all possible configurations of a given number of transmitters as identical, thus leading to the same result.

\subsection{Comparison with 802.11n in Mobile Environment}

This section finally compares the throughput achievable by the TAMPC and SAMPC schemes with that of the $802.11 \mathrm{n}$ extension in a mobile environment. The $802.11 \mathrm{n}$ amendment is implemented using a Vertical Bell Laboratories Layered Space-Time (VBLAST) aided PHY layer for achieving an increased data rate through spatial multiplexing [7]. To this purpose, it is worth noticing that the presented protocols and the 802.11n extension differ in two fundamental aspects. First, the $802.11 \mathrm{n}$ amendment allows a unique communication at a time, while the proposed protocols adopt an MPC approach. Secondly, the 802.11n extension and the presented schemes provide their respective maximum performance in two complementary propagation conditions. More precisely, the TAMPC and SAMPC schemes are designed to operate and achieve their best performance in a low-rank environment, where the angular spread of each signal can be considered small, in order to exploit the interference suppression capabilities of smart antenna systems. Conversely, the $802.11 \mathrm{n}$ extension is designed to operate in a high-rank environment, where the different paths of the signal can be considered independent, in order to exploit the MIMO channel by spatial multiplexing. This implies that the angular spread $\sigma_{\phi}$ of the channel has a fundamental role in determining the performance of the different schemes, since it affects the statistic of the DOA for the TAMPC and SAMPC schemes and, being related to the spatial correlation between the signal replicas, it also affects the performance of the VBLAST algorithm. In particular, for the TAMPC and SAMPC protocols, $\sigma_{\phi}$ is simulated using a Laplacian probability density function for the statistic of the

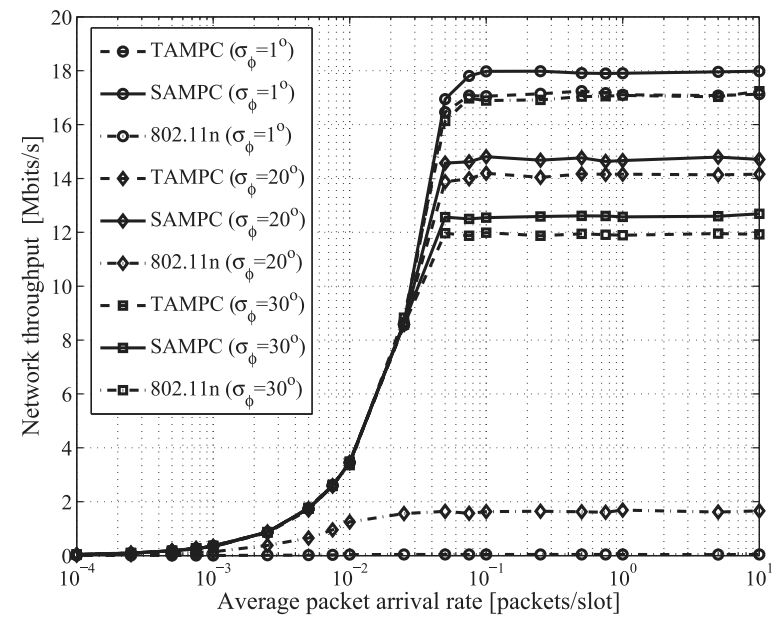

Fig. 8. Network throughput of the TAMPC, SAMPC, and $802.11 \mathrm{n}$ schemes as a function of the average arrival rate for different angular spread values in the presence of block Rayleigh fading.

DOA [53]. For the VBLAST aided 802.11n scheme, the model of [54], which provides a relationship between angular spread and spatial correlation, is used to correlate the signals arriving from the transmitting antennas at a particular receiving antenna. Signals that arrive at different receive antennas are assumed to be uncorrelated, hence the performance of the simulated 802.11n scheme is optimistic. Furthermore, since the TAMPC and SAMPC schemes operate on two channels (CC and MCC) and use LDPC codes, the $802.11 \mathrm{n}$ nodes are fairly allowed adopting the dual-band option at $40 \mathrm{MHz}$ and using LDPC codes [7]. The performance of this 802.11n PHY layer is simulated by transmitting a packet, operating a soft decoding of the received symbols using the minimum mean square error (MMSE)based successive interference cancellation algorithm, and then measuring the mutual information of the sequence of log-likelihood ratios (LLRs). The packet is considered to be successfully received if the mutual information of the sequence of LLRs is higher than the adopted code rate [55]. Mobility is modeled using a block Rayleigh fading channel, which has been considered suitable for the application domain of the compared schemes, involving ad-hoc and distributed mesh networks, in which, differently from vehicular networks, the nodes are characterized by low speeds of movement. Hence, slow fading conditions are assumed, and the modifications of the topology during a single four-way handshake, which identifies a block, are considered negligible. The channel coefficients do not change during a single handshake and are independently generated for different handshakes. The RSSI estimation used by the SAMPC scheme is assumed to be equal to the average received power. Concerning the operations carried out by the TAMPC and SAMPC protocols, it is worth to remark that these operations guarantee the update of the DOAs in the presence of mobility. In fact, when a transmission on the MCC is sensed, the TAMPC and the SAMPC schemes estimate the DOA and receive the packet if this DOA is not present in the NCT or refers to a NAV equal to zero, thus allowing the correct association between the new DOA and the ID of the sensed node.

Fig. 8 reports the throughput of the three schemes obtained using a minimum contention window equal to 32 
for $\sigma_{\phi}=1^{\circ}, 20^{\circ}, 30^{\circ}$. Observe that, differently from the previous figures, the throughput is now reported in Mbits/s in order to reliably compare the $802.11 \mathrm{n}$ protocol, characterized by a single communication at a high data rate, with the proposed MPC schemes, characterized by many communications at a lower data rate. Fig. 8 shows that, in a low-rank environment, the TAMPC and SAMPC protocols maintain an acceptable performance even in the presence of high values of the angular spread, such as $\sigma_{\phi}=20^{\circ}$ and $\sigma_{\phi}=30^{\circ}$, which can be considered realistic for many outdoor scenarios [53]. As expected, the throughput of the VBLAST aided 802.11n scheme becomes significant when the angular spread is sufficiently large to guarantee a reduction of the spatial correlation between the signal replicas. Thus, the TAMPC and SAMPC schemes are able to operate in propagation environments complementary to those usually necessary for MIMO operations (spatial multiplexing and diversity), and, furthermore, the two proposed solutions provide an acceptable throughput when the spatial channel conditions move towards high-rank characteristics.

\section{Conclusions}

This paper has discussed the design requirements for enabling multi-packet communication in IEEE 802.11 networks by using advanced antenna systems, proposing two novel MAC protocols, called TAMPC and SAMPC, which are suitable for asynchronous operations in distributed and heterogeneous scenarios, where legacy and non-legacy nodes equipped with different antenna systems can coexist.

The results have proved that the developed schemes outperform the legacy 802.11 MAC layer with the super-g PHY layer extension. In particular, the SIR-based access adopted by the SAMPC scheme can guarantee higher throughput and fairness with respect to the threshold-based access adopted by the TAMPC protocol, at the cost of an increased but acceptable computational burden. A comparison between the throughput of the TAMPC scheme and that obtained from the theory has shown that the selection of the load threshold has a significant impact on the final performance. Furthermore, simulations have revealed that the adoption of a channel encoder with a code rate not far from unity can be sufficient to maintain a satisfactory performance in different MPC scenarios. In general, both presented protocols, which have been designed to be backward compatible with the 802.11 standard, provide significant throughput values in a low-rank environment, where the MIMO approach adopted by the 802.11n extension may suffer from a performance downgrade due to a large spatial correlation between the signal replicas.

This work aimed to present some novel ideas to deal with the possible forthcoming scenarios involving distributed wireless networks, also providing exhaustive numerical simulations. The subsequent step of the presented activity concerns the development of an experimental testbed, which represents the objective of the current research efforts, in order to test the proposed solutions in a real environment. The here discussed MPC concept has been introduced with the constructive purpose of providing a point of view complementary to the approach adopted by the 802.11ac multipacket extension that has been recently finalized.

\section{ACKNOWLEDGMENTS}

Fulvio Babich is the corresponding author. This work is partly supported by projects GATECOM (Gateway-based architecture for content-centric community networking, Italian University and Research Ministry) and FRA 2013 (Multi-packet communication in 802.11x heterogeneous mobile networks: models and antenna system algorithms).

\section{References}

[1] WLAN MAC/PHY Specific., IEEE 802.11 Std, 1997.

[2] A. Spyropoulos and C. S. Raghavendra, "Asymptotic capacity bounds for ad-hoc networks revisited: The directional and smart antenna cases," in Proc IEEE Global Telecommun. Conf., 2003, pp. 1216-1220.

[3] S. Yi, Y. Pei, and S. Kalyanaraman, "On the capacity improvement of Ad Hoc wireless networks using directional antennas," in Proc. 4th ACM Int. Symp. Mobile Ad Hoc Netw. Comput., 2003, pp. 108-116.

[4] P. Li, C. Zhang, and Y. Fang, "The capacity of wireless Ad Hoc networks using directional antennas," IEEE Trans. Mobile Comput., vol. 10, no. 10, pp. 1374-1387, Oct. 2011.

[5] S. Toumpis and A. J. Goldsmith, "Capacity regions for wireless Ad Hoc networks," IEEE Trans. Wireless Commun., vol. 2, no. 4, pp. 736-748, Jul. 2003.

[6] B. Hamdaoui and K. G. Shin, "Throughput behavior in multihop multiantenna wireless networks," IEEE Trans. Mobile Comput., vol. 8, no. 11, pp. 1480-1494, Nov. 2009.

[7] Enhancements for Higher Throughput, IEEE 802.11n Std, 2009.

[8] L. Tong, Q. Zhao, and G. Mergen, "Multipacket reception in random access wireless networks: From signal processing to optimal medium access control," IEEE Commun. Mag., vol. 39, no. 11, pp. 108-112, Nov. 2001.

[9] L.-C. Wang, S.-Y. Huang, and A. Chen, "On the throughput performance of csma-based wireless local area network with directional antennas and capture effect: A cross-layer analytical approach," in Proc. IEEE Wireless Commun. Netw. Conf., 2004, pp. 1879-1884.

[10] M. M. Carvalho and J. J. Garcia-Luna-Aceves, "Modeling wireless ad hoc networks with directional antennas," in Proc. IEEE INFOCOM, 2006, pp. 1-12.

[11] F. Babich and M. Comisso, "Throughput and delay analysis of 802.11-based wireless networks using smart and directional antennas," IEEE Trans. Commun., vol. 57, no. 5, pp. 1413-1423, May 2009.

[12] D. S. Chan and T. Berger, "Performance and cross-layer design of CSMA for wireless networks with multipacket reception," in Proc. Conf. Rec. 38th Asilomar Conf. Signals, Syst. Comput., 2004, pp. 1917-1921.

[13] F. Babich and M. Comisso, "Theoretical analysis of asynchronous multi-packet reception in 802.11 networks," IEEE Trans. Commun., vol. 58, no. 6, pp. 1782-1794, Jun. 2010.

[14] F. Babich, M. Comisso, A. Crismani, and A. Dorni, "Multi-packet communication in 802.11 networks by spatial reuse: From theory to protocol," in Proc. IEEE Int. Conf. Commun., 2013, pp. 5105-5109.

[15] F. Babich and M. Comisso, "Multi-packet communication by spatial reuse: Application scenarios and open research issues," in Proc. IEEE 9th Int. Wireless Commun. Mobile Comput. Conf., 2013, pp. 1308-1313.

[16] F. Babich and M. Comisso, "Multi-packet communication in heterogeneous wireless networks adopting spatial reuse: Capture analysis," IEEE Trans. Wireless Commun., vol. 12, no. 10, pp. 53465359, Oct. 2013.

[17] A. Zanella and M. Zorzi, "Theoretical analysis of the capture probability in wireless systems with multiple packet reception capabilities," IEEE Trans. Commun., vol. 60, no. 4, pp. 1058-1071, Apr. 2012.

[18] J. A. Stine, "Exploiting smart antennas in wireless mesh networks using contention access," IEEE Wireless Commun., vol. 13, no. 2, pp. 38-49, Apr. 2006.

[19] G. Bianchi, D. Messina, L. Scalia, and I. Tinnirello, "A spacedivision time-division multiple access scheme for high throughput provisioning in WLANs," in Proc. IEEE Int. Conf. Commun., 2005, pp. 2728-2733.

[20] X. Liu, A. Sheth, M. Kaminsky, K. Papagiannaki, S. Seshan, and P. Steenkiste, "DIRC: Increasing indoor wireless capacity using directional antennas," in Proc. ACM SIGCOMM Conf. Data Commun., 2009, pp. 171-182. 
[21] K. Tan, H. Liu, J. Fang, W. Wang, J. Zhang, M. Chen, and G. M. Voelker, "SAM: Enabling practical spatial multiple access in wireless LAN," in Proc. 15th Annu. Int. Conf. Mobile Comput. Netw., 2009, pp. 49-60.

[22] X. Liu, A. Sheth, M. Kaminsky, K. Papagiannaki, S. Seshan, and P. Steenkiste, "Pushing the envelope of indoor wireless spatial reuse using directional access points and clients," in Proc. 16th Annu. Int. Conf. Mobile Comput. Netw., 2010, pp. 209-220.

[23] S. Barghi, H. Jafarkhani, and H. Yousefizadeh, "MIMO-assisted MPR-Aware MAC design for asynchronous WLANs," IEEE/ACM Trans. Netw., vol. 19, no. 6, pp. 1652-1665, Dec. 2011.

[24] D. Lal, R. Toshniwal, R. Radhakrishnan, D. P. Agrawal, and J. Caffery, "A novel MAC layer protocol for space division multiple access in wireless ad hoc networks," in Proc. IEEE 11th Int. Conf. Comput. Commun. Netw., 2002, pp. 614-619.

[25] W.-L. Shen, Y.-C. Tung, K.-C. Lee, K.C.-J. Lin, S. Gollakota, D. Katabi, and M.-S. Chen, "Rate adaptation for 802.11 multiuser MIMO networks," in Proc. 18th Annu. Int. Conf. Mobile Comput. Netw., 2012, pp. 29-40.

[26] J. Yang, J. Li, and M. Sheng, "MAC protocol for mobile ad hoc network with smart antennas," IET Electron. Lett., vol. 39, no. 6, pp. 555-557, Mar. 2003.

[27] N.-S. Fahmy and T.-D. Todd, "A selective CSMA protocol with cooperative nulling for ad hoc networks with smart antennas," in Proc. IEEE Wireless Commun. Netw. Conf., 2004, pp. 387-392.

[28] H. Singh and S. Singh, "Smart-802.11b MAC protocol for use with smart antennas," in Proc. IEEE Int. Conf. Commun., 2004, pp. 36843688.

[29] K. Sundaresan, R. Sivakumar, M. A. Ingram, and T.-Y. Chang, "Medium access control in Ad hoc networks with MIMO links: Optimization considerations and algorithms," IEEE Trans. Mobile Comput., vol. 3, no. 4, pp. 350-365, Oct./Dec. 2004.

[30] R.-R. Choudhury, X. Yang, R. Ramanathan, and N. H. Vaidya, "On designing MAC protocols for wireless networks using directional antennas," IEEE Trans. Mobile Comput., vol. 5, no. 5, pp. 477-491, May 2006.

[31] J. C. Mundarath, P. Ramanathan, and B. D. Van Veen, "A cross layer scheme for adaptive antenna array based wireless ad hoc networks in multipath environments," ACM Wireless Netw., vol. 13, no. 5, pp. 597-615, Oct. 2007.

[32] T. Korakis, G. Jakllari, and L. Tassiulas, "CDR-MAC: A protocol for full exploitation of directional antennas in ad hoc wireless networks," IEEE Trans. Mobile Comput., vol. 7, no. 2, pp. 145-155, Feb. 2008

[33] M. Takai, J. Martin, A. Ren, and R. Bagrodia, "Directional virtual carrier sensing for directional antennas in mobile ad hoc networks," in Proc. 3rd ACM Int. Symp. Mobile Ad Hoc Netw. Comput., 2002, pp. 183-193.

[34] F. Babich, M. Comisso, and A. Dorni, "A PHY design for asynchronous multi-packet reception in 802.11 heterogeneous networks," in Proc. IEEE 73rd Veh. Technol. Conf., 2011, pp. 1-5.

[35] K. C.-J. Lin, S. Gollakota, and D. Katabi, "Random access heterogeneous MIMO networks," in Proc. ACM SIGCOMM Conf., 2011, pp. 146-157.

[36] Enhancements for Very High Throughput for Operation in Bands Below $6 \mathrm{GHz}$, IEEE 802.11ac Std, 2014

[37] F. Babich, M. Comisso, and A. Dorni, "Multi-packet communication in 802.11 networks: A MAC/PHY backward compatible solution," in Proc. IEEE Global Telecomun. Conf., 2011 pp. 1-5.

[38] F. Babich, M. Comisso, and A. Dorni, "A novel sir-based access scheme for multi-packet communication in 802.11 networks," in Proc. IEEE Int. Conf. Commun., 2012, pp. 4494-4498.

[39] L. C. Godara, "Application of antenna arrays to mobile communications, Part II: Beam forming and direction-of-arrival considerations," Proc. IEEE, vol. 85, no. 8, pp. 1193-1245, Aug. 1997.

[40] A. K. Skrivervik, J. F. Zurcher, O. Staub, and J. R. Mosig, "PCS antenna design: The challenge of miniaturization," IEEE Antennas Propag. Mag., vol. 43, no. 4, pp. 12-27, Aug. 2001.

[41] F. Babich, "On the performance of efficient coding techniques over fading channels," IEEE Trans. Wireless Commun., vol. 3, no. 1, pp. 290-299, Jan. 2004.

[42] K. Srinivasan and P. Levis, "RSSI is under appreciated," in Proc. 3rd Workshop Embedded Netw. Sensors, 2006.

[43] “CEVA-XC4500," last access: Aug. 19th, 2014 [Online]. Available: http://www.ceva-dsp.com/CEVA-XC4500
[44] "Qualcomm Hexagon v5," last access: Aug. 19th, 2014 [Online]. Available: https://developer.qualcomm.com/mobiledevelopment/maximize-hardware/multimedia-optimizationhexagon-sdk/tools-and-resources

[45] “TI TMS320C6654," last access: Aug. 19th, 2014. [Online]. Available: http:/ / www.ti.com/product/tms320c6654

[46] Y. Zhang and B. P. Ng, "MUSIC-like doa estimation without estimating the number of sources," IEEE Trans. Signal Process., vol. 58, no. 3, pp. 1668-1676, Mar. 2010.

[47] S.-S. Mahant-Shetti, S. Hosur, and A. Gatherer, "The log-log LMS algorithm," in Proc. IEEE Int. Conf. Acoust., Speech, Signal Process., 1997 pp. 2357-2360.

[48] K. Slattery and H. Skinner, Platform Interference in Wireless Systems: Models, Measurement, and Mitigation. New York, NY, USA: Elsevier Newnes, 2008.

[49] F. Babich and M. Comisso, "Capture analysis of mobile multipacket networks adopting spatial reuse: An alternative study," in Proc. IEEE Global Telecommun. Conf., 2014, pp. 4314-4319.

[50] “IT++ 4.2.0," last access: Aug. 19th, 2014 [Online]. Available: http:/ / sourceforge.net/projects/itpp/files/itpp/4.2.0/

[51] "TL-WN660G: Super G and eXtended Range 108M Wireless mini PCI Adapter," last access: Aug. 19th, 2014 [Online]. Available: http://www.mantronic.com/Products/Networking/tl-wn660g. htm

[52] "OpenWrt: Table of Hardware," last access: Aug. 19th, 2014 [Online]. Available: http://wiki.openwrt.org/toh/start

[53] K. I. Pedersen, P. E. Mogensen, and B. H. Fleury, "A stochastic model of the temporal and azimuthal dispersion seen at the base station in outdoor propagation environments," IEEE Trans. Veh. Technol., vol. 49, no. 2, pp. 437-447, Mar. 2000.

[54] L. C. Tran, T. A. Wysocki, A. Mertins, and J. Seberry, "A generalized algorithm for the generation of correlated rayleigh fading envelopes in wireless channels," EURASIP J. Wireless Commun. Netw., vol. 2005, no. 5, pp. 801-815, Oct. 2005.

[55] M. C. Valenti, "An information-theoretic approach to accelerated simulation of hybrid-ARQ systems," in Proc. IEEE Int. Conf. Commun., 2011, pp. 1-6.

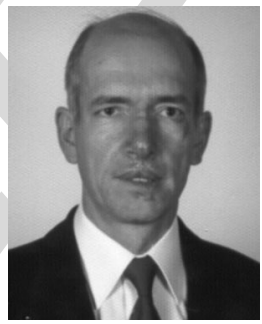

Fulvio Babich received the doctoral degree in electrical engineering, from the University of Trieste, Italy, in 1984. He was at Telettra and worked on optical communications and with Zeltron (Electrolux group) as a company head in the Home System European projects. Currently, he is an associate professor of digital communications in the Department of Engineering and Architecture, University of Trieste. He has served as a co-chair at ICC 2005 and 2014 (Communication Theory Symposia), ICC 2011, and WCSP 2012 (Wireless Communication Symposia). He is a senior member of the IEEE and has been a member of the Directive Board of CNIT (National Inter-University Consortium for Telecommunications, a nonprofit Consortium among 37 Universities for coordinating and fostering basic and applied research).

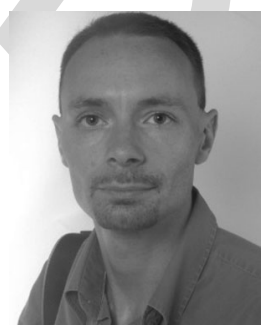

Massimiliano Comisso received the Laurea and the $\mathrm{PhD}$ degrees in information engineering from the University of Trieste, Italy. He worked at Alcatel on optical communications and collaborated with Danieli Automation on electromagnetic sensors' modeling. Currently, he is an assistant professor in the Department of Engineering and Architecture, University of Trieste. His research interests involve distributed networks, antenna array synthesis, and small antennas. $\mathrm{He}$ is a member of the IEEE. 


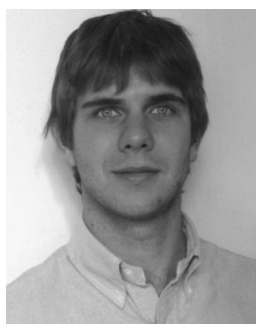

Alessandro Crismani received the master's (summa cum laude) and the PhD degrees in information engineering from the University of Trieste, Italy. He was a visiting student in the Communications Research Group at the University of Southampton, United Kingdom, and a researcher in the Mobile Group of the Networked and Embedded Systems at the University of Klagenfurt, Austria. Currently, he is at u-blox working on $4 \mathrm{G}$ communication systems.

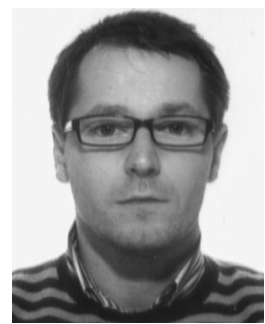

Aljoša Dorni received the master's and $\mathrm{PhD}$ degrees in information engineering from the University of Trieste, Italy. He was a visiting student at the Telecommunications Research Center (FTW) in Vienna, Austria. Currently, he is at ublox working on LTE cellular systems. His research studies focus on distributed wireless networks, embedded systems, network simulators, and software-defined radio platforms. $\mathrm{He}$ is a member of the IEEE.

$>$ For more information on this or any other computing topic, please visit our Digital Library at www.computer.org/publications/dlib. 